\title{
AI Methods in Algorithmic Composition: A Comprehensive Survey
}

\author{
Jose David Fernández \\ Francisco Vico \\ Universidad de Málaga, Calle Severo Ochoa, 4, 119 \\ Campanillas, Málaga, 29590 Spain
}

\author{
JOSEDAVID@GEB.UMA.ES \\ FJV@GEB.UMA.ES
}

\begin{abstract}
Algorithmic composition is the partial or total automation of the process of music composition by using computers. Since the 1950s, different computational techniques related to Artificial Intelligence have been used for algorithmic composition, including grammatical representations, probabilistic methods, neural networks, symbolic rule-based systems, constraint programming and evolutionary algorithms. This survey aims to be a comprehensive account of research on algorithmic composition, presenting a thorough view of the field for researchers in Artificial Intelligence.
\end{abstract}

\section{Introduction}

Many overly optimistic, but ultimately unfulfilled predictions were made in the early days of Artificial Intelligence, when computers able to pass the Turing test seemed a few decades away. However, the field of Artificial Intelligence has grown and got matured, developing from academic research and reaching many industrial applications. At the same time, key projects and challenges have captivated public attention, such as driverless cars, natural language and speech processing, and computer players for board games.

The introduction of formal methods have been instrumental in the consolidation of many areas of Artificial Intelligence. However, this presents a disadvantage for areas whose subject matter is difficult to define in formal terms, which naturally tend to become marginalized. That is the case of Computational Creativity (also known as Artificial Creativity), which can be loosely defined as the computational analysis and/or synthesis of works of art, in a partially or fully automated way. Compounding the problem of marginalization, the two communities naturally interested in this field (AI and the arts) speak different languages (sometimes very different!) and have different methods and goals ${ }^{1}$, creating great difficulties in the collaboration and exchange of ideas between them. In spite of this, small and sometimes fragmented communities are active in the research of different aspects of Computational Creativity.

The purpose of this survey is to review and bring together existing research on a specific style of Computational Creativity: algorithmic composition. Interpreted literally, algorithmic composition is a self-explanatory term: the use of algorithms to compose music. This is a very broad definition, because for centuries musicians have been proposing methods that can be considered as algorithmic in some sense, even if human creativity plays a key

1. Related to this problem, it is not uncommon for engineering concepts to become bent in strange ways when interpreted by artists. See Footnote 28 in page 550 for a particularly remarkable example. 
role. Some commonly cited examples include d'Arezzo's Micrologus, species counterpoint, Mozart's dice games, Schoenberg's twelve-tone technique, or Cage's aleatoric music. Readers interested in these and other pre-computer examples of algorithmic composition are referred to the introductory chapters of almost any thesis or book on the subject, such as Díaz-Jerez's (2000), Aschauer's (2008) or Nierhaus's (2009). In this survey, we will use the term algorithmic composition in a more restricted way, as the partial or total automation of music composition by formal, computational means. Of course, pre-computer examples of algorithmic composition can be implemented on a computer, and some of the approaches reviewed in this survey implement a classical methodology. In general, the focus will be on AI techniques, but self-similarity and cellular automata will also be reviewed as modern computational techniques that can be used for generating music material without creative human input.

\subsection{Motivation}

Some useful starting points for researching the past and present of computer music are the Computer Music Journal, the International Computer Music Conference ${ }^{2}$ annually organized by the International Computer Music Association ${ }^{3}$, and some books such as Machine Models of Music (Schwanauer \& Levitt, 1993), Understanding music with AI (Balaban et al., 1992), Music and Connectionism (Todd \& Loy, 1991), and the anthologies of selected articles from the Computer Music Journal (Roads \& Strawn, 1985; Roads, 1992). However, these resources are not only about algorithmic composition, but computer music in general. For more specific information on algorithmic composition, surveys are a better option.

There are many surveys reviewing work on algorithmic composition. Some review both analysis and composition by computer with AI methods (Roads, 1985), while others discuss algorithmic composition from a point of view related to music theory and artistic considerations (Collins, 2009), or from the personal perspective of a composer (Langston, 1989; Dobrian, 1993; Pope, 1995; Maurer, 1999). Some of them provide an in depth and comprehensive view of a specific technique for algorithmic composition, as Anders and Miranda (2011) do for constraint programming, as Ames (1989) does for Markov chains, or as Santos et al. (2000) do for evolutionary techniques, while some others are specialized in the comparison between paradigms for computational research on music, as Toiviainen (2000). Others offer a wide-angle (but relatively shallow) panoramic of the field (Papadopoulos \& Wiggins, 1999), review the early history of the field (Loy \& Abbott, 1985; Ames, 1987; Burns, 1994), or analyze methodologies and motivations for algorithmic composition (Pearce et al., 2002). There are also works that combine in depth and comprehensive reviews for a wide range of methods for algorithmic composition, such as Nierhaus's (2009) book.

In this context, a natural question arises: why yet another survey? The answer is that no existing survey article fulfills the following criteria: (a) to cover all methods in a comprehensive way, but from a point of view primarily focused on AI research, and (b) to be centered on algorithmic composition. ${ }^{4}$ Nierhaus's (2009) book on algorithmic

2. The archives are available at http://quod.lib.umich.edu/i/icmc/

3. http://www. computermusic.org/

4. Many surveys conflate the discussion on algorithmic composition (synthesis of music) with the computational analysis of music, as in the work of Roads (1985), Nettheim (1997) or Toiviainen (2000). This can become somewhat distracting if the reader is interested just in algorithmic composition. 
composition comes close to fulfilling these criteria with long, detailed expositions for each method and comprehensive reviews of the state of the art. In contrast, this survey is intended to be a reasonably short article, without lengthy descriptions: just a reference guide for AI researchers. With these aims in mind, this survey is primarily structured around the methods used to implement algorithmic composition systems, though early systems will also be reviewed separately.

A second, more practical motivation is accessibility. Since Computational Creativity balances on the edge between AI and the arts, the relevant literature is scattered across many different journals and scholarly books, with a broad spectrum of topics from computer science to music theory. As a very unfortunate consequence, there are many different paywalls between researchers and relevant content, translating sometimes into a lot of hassle, only partially mitigated by relatively recent trends like self-archiving. This survey brings together a substantial body of research on algorithmic composition, with the intention of conveying it more effectively to AI researchers.

\section{Introducing Algorithmic Composition}

Traditionally, composing music has involved a series of activities, such as the definition of melody and rhythm, harmonization, writing counterpoint or voice-leading, arrangement or orchestration, and engraving (notation). Obviously, this list is not intended to be exhaustive or readily applicable to every form of music, but it is a reasonable starting point, especially for classical music. All of these activities can be automated by computer to varying degrees, and some techniques or languages are more suitable for some of these than others (Loy \& Abbott, 1985; Pope, 1993).

For relatively small degrees of automation, the focus is on languages, frameworks and graphical tools to provide support for very specific and/or monotone tasks in the composition process, or to provide raw material for composers, in order to bootstrap the composition process, as a source of inspiration. This is commonly known as computer-aided algorithmic composition (CAAC), and constitutes a very active area of research and commercial software development: many software packages and programming environments can be adapted to this purpose, such as SuperCollider (McCartney, 2002), Csound (Boulanger, 2000), MAX/MSP (Puckette, 2002), Kyma (Scaletti, 2002), Nyquist (Simoni \& Dannenberg, 2013) or the AC Toolbox (Berg, 2011). The development of experimental CAAC systems at the IRCAM $^{5}$ (such as PatchWork, OpenMusic and their various extensions) should also be emphasized (Assayag et al., 1999). Ariza's comprehensive repository of software tools and research resources for algorithmic composition ${ }^{6}$ constitutes a good starting point (Ariza, 2005a) to explore this ecosystem, as well as algorithmic composition in general. Earlier surveys (such as Pennycook, 1985 and Pope, 1986) are also useful for understanding the evolution of the field, especially the evolution of graphical tools to aid composers.

Our survey, on the other hand, is more concerned with algorithmic composition with higher degrees of automation of compositional activities, rather than typical CAAC. In other words, we focus more on techniques, languages or tools to computationally encode human musical creativity or automatically carry out creative compositional tasks with minimal or

5. http://www.ircam.fr/

6. http://www.flexatone.net/algoNet/ 
no human intervention, instead of languages or tools whose primary aim is to aid human composers in their own creative processes.

Obviously, the divide between both ends of the spectrum of automation (CAAC representing a low degree of automation, algorithmic composition a high degree of automation) is not clear, because any method that automates the generation of creative works can be used as a tool to aid composers, and systems with higher degrees of automation can be custombuilt on top of many CAAC frameworks. ${ }^{7}$ Furthermore, a human composer can naturally include computer languages and tools as an integral part of the composition process, such as Brian Eno's concept of generative music (Eno, 1996). To conclude these considerations, this survey is about computer systems for automating compositional tasks where the user is not expected to be the main source of creativity (at most, the user is expected to set parameters for the creative process, encode knowledge about how to compose, or to provide examples of music composed by humans to be processed by the computer). This also includes real-time automatic systems for music improvisation, such as in jazz performance, or experimental musical instruments that automate to a certain extent the improvisation of music.

Finally, a few more considerations, to describe what this survey is not about:

- Although music can be defined as "organized sound", a composition written in traditional staff notation does not fully specify how the music actually sounds: when a piece of music is performed, musicians add patterns of small deviations and nuances in pitch, timing and other musical parameters. These patterns account for the musical concept of expressiveness or gesture, and they are necessary for the music to sound natural. While the problem of automatically generating expressive music is important in itself, and involves creativity, it is clearly not within the boundaries of algorithmic composition as reviewed in this survey. The reader is referred to Kirke and Miranda's (2009) review of this area for further information.

- The computational synthesis of musical sounds, or algorithmic sound synthesis, can be understood as the logical extension of algorithmic composition to small timescales; it involves the use of languages or tools for specifying and synthesizing sound waveforms, rather than the more abstract specification of music associated with traditional staff notation. The line between algorithmic composition and algorithmic sound synthesis is blurred in most of the previously mentioned CAAC systems, but this survey is not concerned with sound synthesis; interested readers may refer to Roads's (2004) book on the subject.

- In computer games (and other interactive settings), music is frequently required to gracefully adapt to the state of the game, according to some rules. This kind of music is commonly referred to as non-linear music (Buttram, 2003) or procedural audio (Farnell, 2007). Composing non-linear music presents challenges of its own, not specifically related to the problem of algorithmic composition, so we will not review the literature on this kind of music.

7. This is the case of many of the systems for algorithmic composition described here. For example, PWConstraints (described in Section 3.2.3) is built on top of PatchWork, as described by Assayag et al. (1999). 
These three scenarios (automated expressiveness, algorithmic sound synthesis and nonlinear music) will be sparingly mentioned in this survey, only mentioned when innovative (or otherwise notable) techniques are involved.

\subsection{The Early Years}

In this section, we will review early research published on algorithmic composition with computers, or with a clear computational approach. While these references might have been discussed by methodology in the following sections, it is useful to group them together here, since it is difficult to find a survey discussing all of them.

The earliest use of computers to compose music dates back to the mid-1950s, roughly at the same time as the concept of Artificial Intelligence was coined at the Darmouth Conference, though the two fields did not converge until some time later. Computers were expensive and slow, and also difficult to use, as they were operated in batch mode.

One of the most commonly cited examples is Hiller and Isaacson's (1958) Illiac Suite, a composition that was generated using rule systems and Markov chains, late in 1956. It was designed as a series of experiments on formal music composition. During the following decade, Hiller's work inspired colleagues from the same university to further experiment with algorithmic composition, using a library of computer subroutines for algorithmic composition written by Baker (also a collaborator of Hiller), MUSICOMP (Ames, 1987). This library provided a standard implementation of the various methods used by Hiller and others.

Iannis Xenakis, a renowned avant-garde composer, profusely used stochastic algorithms to generate raw material for his compositions, using computers since the early 1960s to automate these methods (Ames, 1987). Though his work can be better described as CAAC, he still deserves being mentioned for being a pioneer. Koenig, while not as well known as Xenakis, also was a composer that in 1964 implemented an algorithm (PROJECT1) using serial composition (a musical theory) and other techniques (as Markov chains) to automate the generation of music (Ames, 1987).

However, there were also several other early examples of algorithmic composition, though not so profusely cited as Hiller and Xenakis's. Push Button Bertha, composed in 1956 (Ames, 1987) around the same time as Hiller's Illiac Suite, is perhaps the third most cited example: a song whose music was algorithmically composed as a publicity stunt by Burroughs (an early computer company), generating music similar to a previously analyzed corpus. However, there is at least one earlier, unpublished work by Caplin and Prinz in 1955 (Ariza, 2011), which used two approaches: an implementation of Mozart's dice dame and a generator of melodic lines using stochastic transitional probabilities for various aspects of the composition. Another commonly cited example by Brooks et al. (1957) explored the potential of the Markoff ${ }^{8}$ chain method.

Several other early examples are also notable. Olson's (1961) dedicated computer was able to compose new melodies related to previously fed ones, using Markov processes. While the work was submitted for publication in 1960, they claimed to have built the machine in the early 1950s. Also of interest is Gill's (1963) algorithm, implemented at the request of the

8. "Markov" and "Markoff" are alternative transliterations of the Russian surname Мáрков. The spelling "Markov" has been prevalent for decades, but many older papers used "Markoff". 
$\mathrm{BBC}$, which represents a hallmark in the application of classical AI techniques to algorithmic composition: it used a hierarchical search with backtracking to guide a compositional process inspired by Schoenberg's twelve-tone technique. Finally, it is worth mentioning what may represent the first dissertation on algorithmic composition: Padberg's (1964) Ph.D. thesis implemented a compositional framework (based in formal music theory) in computer code. Her work is unusual in that, instead of using random number generators, she used raw text input to drive procedural techniques in order to generate all the parameters of the composition system.

Non-scholarly early examples also exist, though they are difficult to assess because of the sparsity of the published material, and the fact that they are mostly not peer-reviewed. For example, Pinkerton (1956) described in Scientific American a "Banal Tune-Maker", a simple Markov chain created from several tens of nursery tunes, while Sowa (1956) used a GENIAC machine ${ }^{9}$ to implement the same idea (Cohen, 1962), and Raymond Kurzweil implemented in 1965 (Rennie, 2010) a custom-made device that generated music in the style of classical composers. Another example, unfortunately shrouded in mystery, is Raymond Scott's "Electronium" (Chusid, 1999), an electronic device whose development spanned several decades, reportedly able to generate abstract compositions. Unfortunately, Scott never published or otherwise explained his work.

As machines became less expensive, more powerful and in some cases interactive, algorithmic composition slowly took off. However, aside from the researchers at Urbana (Hiller's university), there was little continuity in research, and reinventing the wheel in algorithmic composition techniques was common. This problem was compounded by the fact that initiatives in algorithmic composition often came from artists, who tended to develop ad hoc solutions, and the communication with computer scientists was difficult in many cases.

\section{The Methods}

The range of methodological approaches used to implement algorithmic composition is notably wide, encompassing many, very different methods from Artificial Intelligence, but also borrowing mathematical models from Complex Systems and even Artificial Life. This survey has been structured by methodology, devoting a subsection to each one:

\subsection{Grammars}

\subsection{Symbolic, Knowledge-Based Systems}

3.3 Markov Chains

\subsection{Artificial Neural Networks}

3.5 Evolutionary and Other Population-Based Methods

\subsection{Self-Similarity and Cellular Automata}

Figure 1 summarizes the taxonomy of the methods reviewed in this survey. Together, Sections 3.1 and 3.2 describe work using symbolic techniques that can be characterized as classical "good old-fashioned AI". Although grammars (Section 3.1) are symbolic and

9. A GENIAC Electric Brain, an electric-mechanic machine promoted as an educational toy. Despite being marketed as a computer device, all the "computing" was performed by the human operator. 


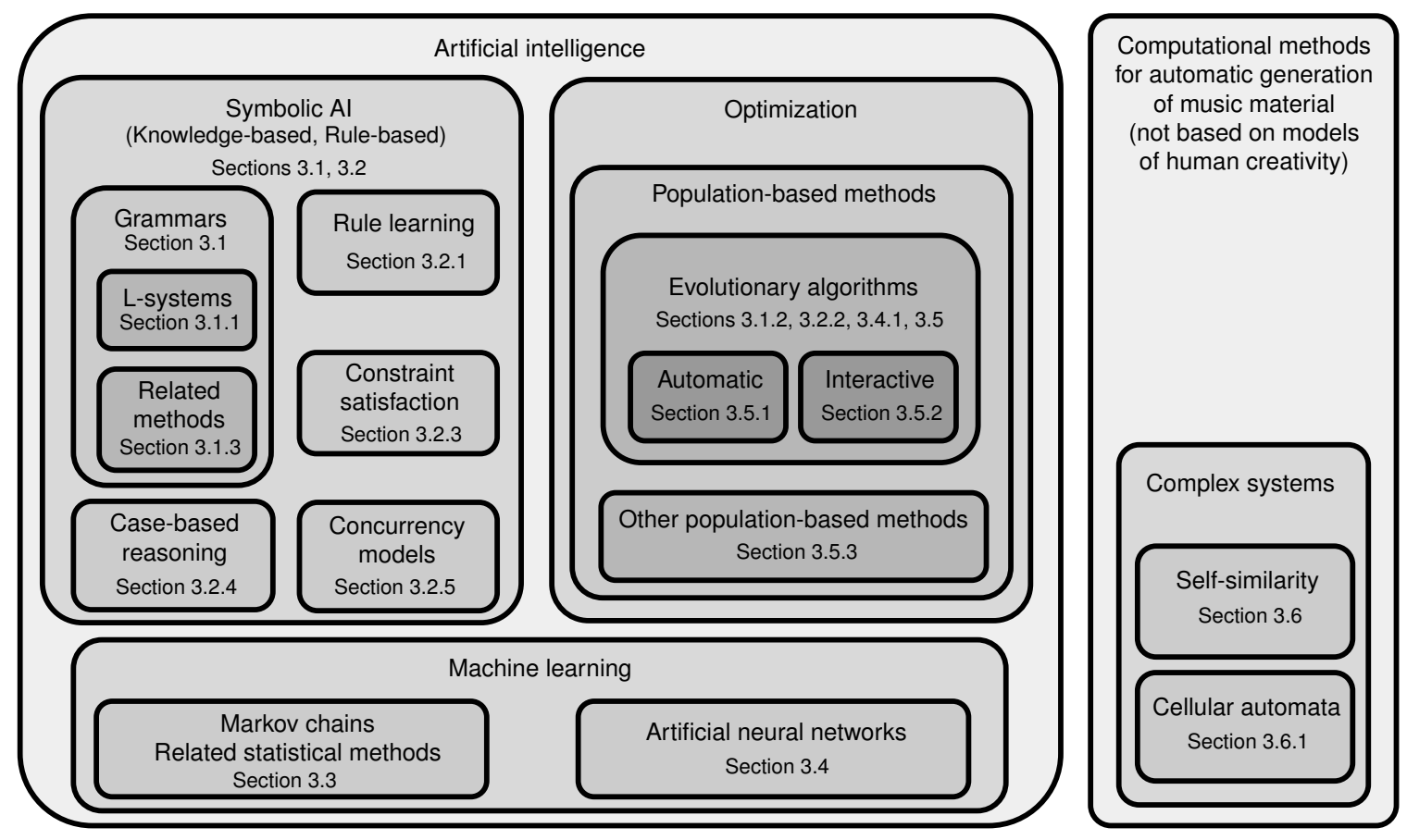

Figure 1: Taxonomy of the methods reviewed in this survey

knowledge-based, and thus should be included as part of Section 3.2, they have been segregated in a separate subsection because of their relative historical importance in algorithmic composition. Sections 3.3 and 3.4 describe work using various methodologies for machine learning, and Section 3.5 does the same for evolutionary algorithms and other populationbased optimization methods. Although the methodologies described in Section 3.6 are not really a form of "Artificial Intelligence", they have been included because of their importance in algorithmic composition as automatic sources of music material (i.e., they do not depend on any model of human creativity for generating music material).

There have been other attempts to systematize algorithmic composition, such as the taxonomies of Papadopoulos and Wiggins (1999) and Nierhaus (2009). Our taxonomy is roughly similar to Nierhaus's, with some differences, such as including L-systems as grammars instead of self-similar systems. The reader may be surprised to find that many methods for machine learning and optimization are missing from our taxonomy. There are several reasons for this. In some cases, some methods are subsumed by others. For example, in machine learning, many different methods have been formulated in the mathematical framework of artificial neural networks. In other cases, a method has been used only rarely, almost always together with other methods. For example, in optimization, this is the case of tabu search, which has been used a few times in the context of constraint satisfaction problems (Section 3.2.3), and simulated annealing, which has been occasionally combined with constraint satisfaction, Markov processes and artificial neural networks.

It is difficult to neatly categorize the existing literature in algorithmic composition with any hierarchical taxonomy, because the methods are frequently hybridized, giving rise to many possible combinations. This is specially true for evolutionary methods, which have 
been combined with almost every other method. Additionally, some papers can be considered to belong to different methodologies, depending on the selected theoretical framework $^{10}$, while others are unique in their approaches ${ }^{11}$, further complicating the issue. Finally, the lines between some methods (as rule systems, grammars and Markov chains) are frequently blurred: in some cases, ascribing a work to one of them becomes, in the end, a largely arbitrary exercise depending on the terminology, intentions and the domain of the researchers. Each method will be presented separately (but also presenting existing hybridizations with other methods), describing the state of the art in a mostly chronological order for each method.

Although this classification is not fully comprehensive, we have only found one (arguably remote) example using a method that is not related to the ones listed above: Amiot et al. (2006), who applied the Discrete Fourier Transform (DFT) to generate variations of musical rhythms. Given a rhythm as a sequence of numerical symbols, they represented it in the frequency domain by computing its DFT. Variations on that rhythm were generated by slightly perturbing the coefficients of the transform and converting back to the time domain.

\subsection{Grammars and Related Methods}

In broad terms, a formal grammar may be defined as a set of rules to expand high-level symbols into more detailed sequences of symbols (words) representing elements of formal languages. Words are generated by repeatedly applying rewriting rules, in a sequence of so-called derivation steps. In this way, grammars are suited to represent systems with hierarchical structure, which is reflected in the recursive application of the rules. As hierarchical structures can be recognized in most styles of music, it is hardly surprising that formal grammar theory has been applied to analyze and compose music for a long time ${ }^{12}$, despite recurring concerns that grammars fail to capture the internal coherency and subtleties required for music composition (Moorer, 1972).

To compose music using formal grammars, an important step is to define the set of rules of the grammar, which will drive the generative process. The rules are traditionally multi-layered, defining several subsets (maybe even separated in distinct grammars) of rules for different phases of the composition process: from the general themes of the composition, down to the arrangement of individual notes. While early authors derived the rules by hand from principles grounded in music theory, other methods are possible, like examining a corpus of pre-existing musical compositions to distill a grammar able to generate compositions in the general style of the corpus, or using evolutionary algorithms. Another important aspect is the mapping between the formal grammar and the musical objects that it generates, which usually relates the symbols of the derived sequences with elements of the music composition, as notes, chords or melodic lines. However, other mappings are possible, as using the derivation tree to define the different aspects of the musical composition. Another important aspect of the automatic composition process is the election of the grammatical

10. For example, Markov chains can be formulated as stochastic grammars; some self-similar systems can be characterized as L-system grammars; rule learning and case-based reasoning are also machine learning methods; etc.

11. For example, Kohonen's method (Kohonen et al., 1991), which is neither grammatical nor neural nor Markovian, but can be framed in either way, according to its creator.

12. See, e.g., the survey by Roads (1979). 


\begin{tabular}{|c|c|c|}
\hline Reference & Composition task & Comments \\
\hline Lidov \& Gabura, 1973 & melody & early proposal \\
\hline Rader, 1974 & melody & $\begin{array}{c}\text { early proposal, } \\
\text { very detailed grammar }\end{array}$ \\
\hline Ulrich, 1977 & jazz chord identification & $\begin{array}{l}\text { integrated in an ad hoc system } \\
\text { (to produce jazz improvisations) }\end{array}$ \\
\hline Baroni \& Jacoboni, 1978 & grammar for Bach chorales & early proposal \\
\hline $\begin{array}{l}\text { Leach \& Fitch, } 1995 \\
\text { (XComposer) }\end{array}$ & structure, rhythm and melody & $\begin{array}{l}\text { uses chaotic non-linear systems } \\
\text { (self-similarity) }\end{array}$ \\
\hline Hamanaka et al., 2008 & $\begin{array}{l}\text { generate variations on two melodies } \\
\text { (by altering the derivation tree) }\end{array}$ & $\begin{array}{l}\text { inspired by Lerdahl et al.'s (1983) } \\
\text { GTTM }\end{array}$ \\
\hline Roads, 1977 & structure, rhythm and melody & grammar compiler \\
\hline Holtzman, 1981 & structure, rhythm and melody & grammar compiler \\
\hline Jones, 1980 & structure & $\begin{array}{c}\text { space grammars } \\
\text { (uses the derivation tree) }\end{array}$ \\
\hline $\begin{array}{c}\text { Bel, } 1992 \\
\text { (Bol Processor) }\end{array}$ & improvisation of tabla rhythms & tool for field research \\
\hline Kippen \& Bel, 1989 & improvisation of tabla rhythms & grammatical inference \\
\hline $\begin{array}{c}\text { Cruz-Alcázar \& Vidal-Ruiz, } \\
1998\end{array}$ & melody & grammatical inference \\
\hline Gillick et al., 2009 & jazz improvisation & $\begin{array}{l}\text { grammatical inference. } \\
\text { Implemented as an extension to } \\
\text { Keller and Morrison's (2007) } \\
\text { ImprovGenerator }\end{array}$ \\
\hline $\begin{array}{l}\text { Kitani \& Koike, } 2010 \\
\text { (ImprovGenerator) }\end{array}$ & real-time drum rhythm improvisation & online grammatical inference \\
\hline $\begin{array}{l}\text { Keller \& Morrison, } 2007 \\
\quad \text { (Impro-Visor) }\end{array}$ & jazz improvisation & sophisticated GUI interface \\
\hline Quick, 2010 & classical three-voice counterpoint & $\begin{array}{l}\text { integrated in a Schenkerian } \\
\text { framework }\end{array}$ \\
\hline Chemillier, 2004 & jazz chord sequences & $\begin{array}{l}\text { implemented in OpenMusic and } \\
\text { MAX }\end{array}$ \\
\hline
\end{tabular}

Table 1: References for Section 3.1 (algorithmic composition with grammars), in order of appearance.

rules to be applied. While many approaches are possible, the use of activation probabilities for the rules (stochastic grammars) is common. In the process of compiling information for this survey, it has been noted that almost all research has been done on regular and context-free grammars, as context-sensitive and more general grammars seem to be very difficult to implement effectively, except for very simple toy systems.

Lidov and Gabura (1973) implemented an early example of a formal grammar to compose simple rhythms. Another early example was implemented by Rader (1974): he defined a grammar by hand from rather simple music concepts, enriching the rules of the grammar with activation probabilities. Other early examples used grammars driven by rules from music theories, either as a small part of a synthesis engine, as Ulrich's (1977) grammar 
for enumerating jazz chords, or by inferring the rules from classical works, as Baroni and Jacoboni's (1978) grammar to generate melodies. A Generative Theory of Tonal Music (Lerdahl et al., 1983), a book presenting a grammatical analysis of tonal music, is a relatively early theoretical work that can be said to have influenced the use of grammars for algorithmic composition, though it is not directly concerned with algorithmic composition, but with a grammatical approach to the analysis of music. This book has been widely popular, and has had a lasting impact on the field and high citation rates. Examples of later work inspired by this book include Pope's (1991) "T-R Trees", Leach and Fitch's (1995) "event trees", and Hamanaka et al.s (2008) "melody morphing".

In the 1980s, some proposed approaches more in line with computer science, abstracting the process to generate the grammars instead of codifying them by hand, though at the cost of producing less interesting compositions. Roads (1977) proposed a framework to define, process and use grammars to compose music, while Holtzman (1981) described a language to define music grammars and automatically compose music from them. Meanwhile, Jones (1980) proposed the concept of space grammars, in conjunction with a novel mapping technique: instead of using the terminal symbols as the building blocks of the composition, he used the derivation tree of the terminal sequence to define the characteristics of the composition. This approach was unfortunately not developed far enough to yield significant results. In spite of these early efforts, most research on grammatical representations of music was focused on analysis rather than synthesis. Some instances, such as Steedman's (1984) influential grammar for the analysis of jazz chord progressions, were later adapted for synthesis (see below).

The problem with a grammatical approach to algorithmic composition is the difficulty to manually define a set of grammatical rules to produce good compositions. This problem can be solved by generating the rules of the grammar (and the way they are applied) automatically. For example, although Bel (1992) implemented the BOL processor to facilitate the creation by hand of more or less sophisticated music grammars ${ }^{13}$, he also explored the automated inference of regular grammars (Kippen \& Bel, 1989). Later, Cruz-Alcázar and Vidal-Ruiz (1998) implemented several methods of grammatical inference: analyze a corpus of pre-existing classical music compositions, represented with a suitable set of symbols, then inducing stochastic regular grammars (Markov chains) able to parse the compositions in the corpus, and finally applying these grammars to generate new compositions that are in a similar style to the compositions in the corpus. Gillick et al. (2009) used a similar approach (also Markovian) to synthesize jazz solos, but with a more elaborated synthesis phase. Kitani and Koike (2010) provide another example of grammatical inference, in this case used for real-time improvised accompaniment.

However, others still designed their grammars by hand, carefully choosing the mapping between terminal symbols and musical objects, as Keller and Morrison (2007) did for jazz improvisations. Another approach is to take a pre-existing music theory with a strong hierarchical methodology, as designing a grammar inspired in Schenkerian analysis (Quick, 2010), or using Lerdhal's grammatical analysis to derive new compositions from two previously existing ones by altering the derivation tree (Hamanaka et al., 2008), or even de-

13. Initially to represent and analyze informal knowledge about Indian tabla drumming, but later also to represent other music styles. 


\begin{tabular}{|c|c|c|}
\hline Reference & Composition task & Comments \\
\hline Prusinkiewicz, 1986 & melody & mapping turtle graphics to music scores \\
\hline Nelson, 1996 & melody & mapping turtle graphics to music scores \\
\hline Mason \& Saffle, 1994 & melody (counterpoint is suggested) & mapping turtle graphics to music scores \\
\hline Soddell \& Soddell, 2000 & $\begin{array}{l}\text { aural representations } \\
\text { of biological data }\end{array}$ & L-system modulates pitch intervals \\
\hline Morgan, 2007 & $\begin{array}{l}\text { composition for a large } \\
\text { instrumental ensemble }\end{array}$ & ad hoc symbolic mapping \\
\hline Langston, 1989 & melody & $\begin{array}{l}\text { L-system is interpreted to arrange } \\
\text { pre-specified fragments }\end{array}$ \\
\hline Worth \& Stepney, 2005 & melody & several mappings and L-system types \\
\hline Manousakis, 2006 & melody (sound synthesis) & $\begin{array}{l}\text { complex, multi-dimensional mapping. } \\
\text { Implemented in MAX. }\end{array}$ \\
\hline McCormack, 1996 & melody, polyphonies & contex-sensitive L-systems \\
\hline DuBois, 2003 & real-time accompaniment & implemented in MAX \\
\hline Wilson, 2009 & melody & mapping turtle graphics to music scores \\
\hline McGuire, 2006 & arpeggiator & simple symbolic mapping \\
\hline Watson, 2008 & base chord progression & $\begin{array}{l}\text { L-systems are used the context of a } \\
\text { larger, multi-stage system }\end{array}$ \\
\hline Gogins, 2006 & voice leading & $\begin{array}{l}\text { Musical theory (pitch spaces). } \\
\text { Implemented in Csound }\end{array}$ \\
\hline Bulley \& Jones, 2011 & arpeggiator & $\begin{array}{c}\text { part of a real-time art installation. } \\
\text { Implemented in MAX }\end{array}$ \\
\hline Pestana, 2012 & real-time accompaniment & implemented in MAX \\
\hline
\end{tabular}

Table 2: References for Section 3.1.1, in order of appearance.

veloping a jazz on-the-fly improviser (Chemillier, 2004) by adapting Steedman's grammar, previously implemented for analysis purposes.

\subsubsection{L-SYSTEMS}

Lindenmayer Systems, commonly abbreviated to L-systems, are a specific variant of formal grammar, whose most distinctive feature is parallel rewriting, i.e., at each derivation step, not one but all possible rewriting rules are applied at once. They have been successfully applied in different scenarios, specially to model microbial, fungi and plant growth and shapes, because they are particularly well-suited to represent the hierarchical self-similarity characteristic of these organisms. This ability to represent self-similar structures, together with the fact that L-systems are easier to understand and apply than traditional formal grammars, have made L-systems fairly popular in algorithmic composition.

Arguably, the most visually stunning way to use L-systems has been the synthesis of $2 \mathrm{D}$ and 3D renderings of plants, using a mapping from sequences of symbols to graphics based on turtle graphics (Prusinkiewicz \& Lindenmayer, 1990). It is only natural that the first application of L-systems to algorithmic composition used turtle graphics to render an image that was then interpreted into a musical score (Prusinkiewicz, 1986), mapping 
coordinates, angles and edge lengths into musical objects. This approach has been used by music composers, as Nelson's (1996) Summer Song and Mason and Saffle's (1994) idea of using different rotations and stretchings of the image to implement counterpoint. As a funny side note, Soddell and Soddell (2000) generated aural renditions of their biological L-system models, to explore new ways to understand them. Additionally, other composers used new approaches not dependent upon the graphical interpretation of the L-systems, such as Morgan's (2007) symbolic mapping. One popular is to pre-generate a collection of short fragments and/or other musical objects, and define an algorithm to interpret the final sequence of symbols as instructions that transform and arrange the fragments into a composition. This approach has been used by Langston (1989) and Kyburz (Supper, 2001), while Edwards (2011) used a more convoluted but ultimately similar mapping.

However, these two approaches (the graphics-to-music and the pre-generated sequences) only scratch the surface of the technical possibilities to generate music with L-systems; many other mappings are possible (Worth \& Stepney, 2005). In some cases, these mappings can become exceedingly complex, such as the implementation of Manousakis (2006), whose L-systems drove a multidimensional automata whose trajectory was then interpreted as music. While most composers and researchers experimented with context-free L-systems, McCormack (1996, 2003a) used context-sensitive, parametric L-systems to increase the expressiveness of the compositions and enable the implementation of polyphony. He also used a rich and comprehensive mapping from the symbol sequence to the musical score, interpreting the symbols in the sequence as instructions to modulate the parameters of an automata driving a MIDI synthesizer, though the grammars were ultimately specified by hand. DuBois (2003) used a simpler but also rich approach, mapping the symbols to elemental musical objects (as notes or instruments) or simple transformations applied to them, using brackets to encode polyphony. He also used L-systems to drive real-time synthetic accompaniment, by extracting features from the audio signal of a performer (as the pitch and loudness of the notes), encoding them as symbols to be expanded by L-system rules, and using the resulting symbol sequences to drive MIDI synthesizers. In spite of these developments, new mappings based on the images rendered by the turtle method are still investigated (Wilson, 2009).

L-systems can also be used to implement tools to assist the compositional process by solving just a part of it, as generating more complex arpeggios than off-the-shelf arpeggiators (McGuire, 2006), or providing just the base chord progression of a composition (Watson, 2008), sometimes applying elements of music theory to implement the rules (Gogins, 2006). Another area of research is the implementation of real-time improvisers, either for limited parts of the composition process (Bulley \& Jones, 2011), or for accompaniment (Pestana, 2012).

\subsubsection{Grammars and Evolutionary Algorithms}

Evolutionary methods have also been used together with grammars. In this case, a common approach is to evolve the grammatical rules, as in GeNotator (Thywissen, 1999), in which the genomes are grammars specified through a GUI and the fitness function is interactive (the user assigns the fitness of the grammars). A more exotic example by Khalifa et al. 


\begin{tabular}{|c|c|c|}
\hline Reference & Composition task & Comments \\
\hline \hline $\begin{array}{c}\text { Thywissen, 1999 } \\
\text { (GeNotator) }\end{array}$ & structure & $\begin{array}{c}\text { the grammar is the genotype } \\
\text { in interactive evolutionary algorithm }\end{array}$ \\
\hline Khalifa et al., 2007 & melody & the grammar is part of the fitness function \\
\hline Ortega et al., 2002 & melody & grammatical evolution \\
\hline Reddin et al., 2009 & melody & interactive grammatical evolution \\
\hline $\begin{array}{c}\text { Shao et al., 2010 } \\
\text { (Jive) }\end{array}$ & melody & interactive evolutionary algorithm with L-systems \\
\hline Bryden, 2006 & melody & interactive evolutionary algorithm with L-systems \\
\hline Fox, 2006 & melody & evolutionary algorithm with L-systems \\
\hline Peck, 2011 & melody & grammatical evolution with L-systems \\
\hline Dalhoum et al., 2008 &
\end{tabular}

Table 3: References for Section 3.1.2, in order of appearance.

(2007) uses the grammar as part of the fitness function instead of the generation of the compositions.

Some evolutionary methods are specifically adapted to handle grammars. This is the case of grammatical evolution, a method in which the genomes are sequences of numbers or symbols controlling the application of rules of a pre-defined (and possibly stochastic) grammar. The most common approach is to represent the music as the output from the grammar, which can range from very general to specific for a given music style. Several instances of this method have been developed: from an early, bare-bones implementation (Ortega et al., 2002) to a more elaborated one using a simple fitness function based on general concepts from music theory (Reddin et al., 2009). However, there are other approaches, such as the system implemented by Shao et al. (2010), whose grammar is used to produce intermediate code, which is then used to generate the music.

As in the more general case of formal grammars, evolutionary algorithms have been used to create L-systems. However, most examples use an interactive fitness function (the fitness is assigned by a human), like the basic implementation of Bryden (2006) and the approach based on genetic programming used by Fox (2006). Others use very simplistic fitness functions, with modest results (Peck, 2011). A more sophisticated approach was used by Dalhoum et al. (2008), using grammatical evolution with a fitness function based on a distance metric of the synthesized compositions to a pre-specified corpus of compositions.

\subsubsection{Related Methods}

Finally, this subsection presents a few examples that do not exactly use grammars, but utilize similar or borderline approaches.

The first one is the application of Kohonen's Dynamically Expanding Context (DEC) method to algorithmic composition (Kohonen et al., 1991). In DEC, a set of music examples is fed to the algorithm, which infers a model from the structure of the examples that may be construed as a stochastic context-sensitive grammar. The model is as parsimonious as possible, that is, the rules have as little contextual information as possible. Then, the inferred grammar is used to generate new compositions. Drewes and Högberg's (2007) 


\begin{tabular}{|c|c|c|}
\hline Reference & Composition task & Comments \\
\hline \hline Kohonen et al., 1991 & melody & Uses Kohonen's Dynamically Expanding Context \\
\hline Drewes \& Högberg, 2007 & $\begin{array}{c}\text { generate variations on } \\
\text { a melody }\end{array}$ & applies tree-based algebraic transformations \\
\hline $\begin{array}{c}\text { Cope, 1992 (EMI), } \\
2000 \text { (SARA, ALICE), }\end{array}$ & melody & EMI uses Augmented Transition Networks \\
\hline
\end{tabular}

Table 4: References for Section 3.1.3, in order of appearance.

work is also borderline, using regular tree grammars to generate a basic scaffold that is then modified by algebraic operations to generate a final music composition.

But the more famous example in this category is Cope's (1992) "Experiments in Musical Intelligence" (EMI), a software application able to analyze a set of musical compositions in a specific style (for example, Bach's) and to derive an Augmented Transition Network (ATN), i.e., a finite state automaton able to parse relatively complex languages. EMI then applies pattern-matching algorithms to extract signatures or short musical sequences characteristic of the style of the set of examples being analyzed, determining how and when to use these signatures in compositions with that style. After this analysis, the synthesis phase generates new music compositions that comply with the specifications encoded in the inferred ATN, with quite impressive results. He iterated EMI's design in other applications, like SARA and ALICE (Cope, 2000), but ultimately tried a new approach with yet another application, "Emily Howell". Cope (2005) reported that "Emily Howell" developed a unique style by a process of trial and error guided by human input; however, other researchers (Wiggins, 2008) have disputed the validity of his methodology.

\subsection{Symbolic, Knowledge-Based Systems and Related Methods}

Here, knowledge-based system is used as an umbrella term encompassing various rule-based systems under several different paradigms, with the common denominator of representing knowledge as more or less structured symbols. Since knowledge about musical composition has traditionally been structured as sets of more or less formalized rules for manipulating musical symbols (Anders \& Miranda, 2011), knowledge-based and rule systems come as a natural way to implement algorithmic composition. In fact, it is extremely common for algorithmic composition systems to include some kind of composition rules at some point of the workflow. The most known early work on algorithmic composition is an example: classical rules for counterpoint were used in the generation of the first and second movements of the Illiac Suite (Hiller \& Isaacson, 1958). Because of this, this subsection is mostly confined to the description of systems with strong foundations in AI (as expert systems), sidestepping to a certain degree the works of composers that are difficult to categorize, because of the ad hoc nature of their approaches and the very different language they use.

Starting with an exposition of early work, Gill's (1963) paper, already cited in Section 2.1, presented the first application of classical AI heuristics to algorithmic composition: he used a hierarchical search with backtracking to guide a set of compositional rules from Schoenberg's twelve-tone technique. Another notable example is Rothgeb's (1968) Ph.D. thesis: he encoded in SNOBOL a set of rules extracted from eighteenth century 


\begin{tabular}{|c|c|c|}
\hline Reference & Composition task & Comments \\
\hline Gill, 1963 & $\begin{array}{l}\text { Schoenberg's twelve-tone } \\
\text { technique }\end{array}$ & hierarchical search with backtracking \\
\hline Rothgeb, 1968 & unfigured bass & implemented in SNOBOL \\
\hline $\begin{array}{l}\text { Thomas, } 1985 \\
\text { (Vivace) }\end{array}$ & four-part harmonization & implemented in LISP \\
\hline $\begin{array}{l}\text { Thomas et al., } 1989 \\
\text { (Cantabile) }\end{array}$ & Indian raga style & implemented in LISP \\
\hline Steels, 1986 & four-part harmonization & uses Minsky's frames \\
\hline $\begin{array}{l}\text { Riecken, } 1998 \\
\text { (Wolfgang) }\end{array}$ & melody & uses Minsky's SOM \\
\hline Horowitz, 1995 & jazz improvisation & uses Minsky's SOM \\
\hline $\begin{array}{c}\text { Fry, } 1984 \\
\text { (Flavors Band) }\end{array}$ & $\begin{array}{l}\text { jazz improvisation and other } \\
\text { styles }\end{array}$ & $\begin{array}{l}\text { phrase processing networks (networks of } \\
\text { agents encoding musical knowledge) }\end{array}$ \\
\hline $\begin{array}{l}\text { Gjerdingen, } 1988 \\
\text { (Praeneste) }\end{array}$ & species counterpoint & implements a theory of how composers work \\
\hline Schottstaedt, 1989 & species counterpoint & constraint-based search with backtracking \\
\hline Löthe, 1999 & piano minuets & set of rules extracted from a classical textbook \\
\hline Ulrich, 1977 & jazz improvisation & also uses a grammar (for jazz chords) \\
\hline Levitt, 1981 & jazz improvisation & $\begin{array}{l}\text { Criticized by Horowitz (1995) } \\
\text { for being overly primitive }\end{array}$ \\
\hline Hirata \& Aoyagi, 1988 & jazz improvisation & uses logic programming \\
\hline $\begin{array}{l}\text { Rowe, } 1992 \\
\text { (Cypher) }\end{array}$ & $\begin{array}{c}\text { interactive jazz } \\
\text { improvisation }\end{array}$ & uses Minsky's SOM \\
\hline $\begin{array}{c}\text { Walker, } 1994 \\
\text { (ImprovisationBuilder) }\end{array}$ & $\begin{array}{c}\text { interactive jazz } \\
\text { improvisation }\end{array}$ & implemented in SmallTalk \\
\hline $\begin{array}{l}\text { Ames \& Domino, } 1992 \\
\text { (Cybernetic Composer) }\end{array}$ & jazz, rock & also uses Markov chains for rhythm \\
\hline
\end{tabular}

Table 5: References for Section 3.2, in order of appearance.

music treatises to harmonize the unfigured bass, that is to say, determine adequate chords from a sequence of bass notes. ${ }^{14}$ He discovered that the classical rules were incomplete and incoherent to a certain extent.

These were recurring problems for many others implementing rules of composition straight from musical theory. For example, Thomas (1985) designed a rule-based system for four-part chorale harmonization implemented in Lisp ${ }^{15}$, with the intent of clarifying the musical rules she taught to her students. Later, she designed another rule system (Thomas et al., 1989) for simple melody generation in the Indian raga style. Another example in harmonization is the use of Minsky's paradigm of frames by one of his students to encode a set of constraints to solve a relatively simple problem from tonal harmony, finding a passing chord between two others (Steels, 1979), and later to tackle the problem of four-part harmonization (Steels, 1986). Minsky developed other paradigms, such as K-lines and the

14. It should be noted that this stems from the practice of not completely specifying the harmonization, a problem that performers were expected to solve by improvisation.

15. All the systems discussed in this paragraph were implemented in Lisp. 


\begin{tabular}{|c|c|c|}
\hline Reference & Composition task & Comments \\
\hline \hline $\begin{array}{c}\text { Schwanauer, 1993 } \\
\text { (MUSE) }\end{array}$ & $\begin{array}{c}\text { four-part } \\
\text { harmonization }\end{array}$ & $\begin{array}{c}\text { presents the learning techniques in a similar way to } \\
\text { Roads (1985, sect. 8.2) }\end{array}$ \\
\hline Widmer, 1992 & harmonization & based on user evaluations of a training corpus \\
\hline Spangler, 1999 & $\begin{array}{c}\text { real-time four-part } \\
\text { harmonization }\end{array}$ & $\begin{array}{c}\text { prioritizes harmonic errors by severity, } \\
\text { in order to refine the results }\end{array}$ \\
\hline Morales \& Morales, 1995 & species counterpoint & uses logic programming \\
\hline
\end{tabular}

Table 6: References for Section 3.2.1, in order of appearance.

Society of Mind (SOM), which also influenced the work on algorithmic composition of two of his students, Riecken and Horowitz. Riecken (1998) used them in a system that composed monophonic melodies according to user-specified "emotional" criteria, while Horowitz (1995) used them in a system that improvised jazz solos. Fry's (1984) phrase processing networks, while not directly based on SOM, were specialized procedural representations of networks of agents implementing musical transformations to encode knowledge about jazz improvisation and other styles.

Other researchers have also explored different ways to generate species counterpoint with rule-based systems: Gjerdingen (1988) implemented a system based on the use of several pre-specified musical schemata, implementing a theory of how composers work, while Schottstaedt (1989) used a more formal approach: a constraint-based search with backtracking. He followed a classical rulebook on species counterpoint, to the point of bending some rules and creating new ones in order to get as close as possible to the scores serving as examples in that book. Also on the formal side, Löthe (1999) extracted a set of rules from a classical textbook for composing minuets.

Other music styles demanded different approaches: as jazz performances are improvisations over existing melodies, knowledge-based systems for jazz were structured as more or less sophisticated analysis-synthesis engines. For example, the work of Ulrich (1977): his system analyzed a melody and fitted it to a harmonic structure. Another student of Minsky (Levitt, 1981) implemented a rule-based jazz improviser formulating some of the rules as constraints, while Hirata and Aoyagi (1988) encoded the rules in logic programming, trying to design a more flexible system. Rowe (1992) used a SOM architecture $^{16}$ for Cypher, an analysis-synthesis engine able to play jazz interactively with a human performer, notable for its flexibility and the musical knowledge encoded into it. Also, Walker (1994) implemented an object-oriented analysis-synthesis engine able to play jazz interactively with a human performer, and Ames and Domino (1992) implemented a hybrid system (using rules and Markov chains) for the generation of music in several popular genres.

\subsubsection{Rule LEARning}

While the knowledge implemented in rule-based systems is usually static, part of the knowledge may be dynamically changed or learned. The natural term for this concept is machine learning, but its meaning is unfortunately vague, because it is used as a catch-all for many methods, including neural networks and Markov chains.

16. He was not a student of Minsky, though. 
A few examples of rule-based learning systems have been developed. For example, Schwanauer (1993) implemented MUSE, a rule-based system for solving several tasks in fourpart harmonization. While the core ruleset was static, a series of constraints and directives for the composition process where also built in the system, and their application was also used to dynamically change the rule priorities. Additionally, when the system successfully solved a task, it was able to deduce new composite rules by extracting patterns of rule application. Widmer (1992) implemented another example: a system for the harmonization of simple melodies. It was based on user evaluations of a training corpus: from a hierarchical analysis of the training melodies and theirs evaluations, it extracted rules of harmonization. Spangler (1999) implemented a system for generating rule systems for harmonizing fourpart chorales in the style of Bach, with the constraint of doing the harmonization in real time. The rulesets were generated by analyzing databases of examples with algorithms that applied formal concepts of information theory for distilling the rules, and the violations of harmonic rules were prioritized in order to refine the results. Using the framework of logic programming, Morales and Morales (1995) designed a system that learned rules of classical counterpoint from musical examples and rule templates.

\subsubsection{Rule-Based Methods and Evolutionary Algorithms}

The most intuitive way to hybridize rule-based knowledge systems and evolutionary algorithms is to craft a fitness function from the ruleset. This can be done efficiently for domains whose rules have been adequately codified, and compliance with the rules can be expressed as a graduated scale, instead of a binary (yes/no) compliance.

A good example is four-part baroque harmonization for a pre-specified melody, which lends itself particularly well to this approach. McIntyre (1994) extracted a set of rules for performing this harmonization from classical works, and codified them as a set of scoring functions. The fitness was a weighted sum of these scores, with a tiered structure: some scores were not added unless other specific scores had values above some thresholds (because they were more critical or prerequisites to produce good harmonizations). A slightly different approach was used by Horner and Ayers (1995): they defined two classes of rules: one for defining acceptable voicings for individual chords, used to enumerate all possible voicings, and another for defining how the voices are allowed to change between successive chords. An evolutionary algorithm was used to find music compositions, whose search space was constructed with the enumeration of voicings (first class of rules). The fitness of each candidate solution was simply the amount of violated rules from the second class. Phon-Amnuaisuk et al. (1999) also did four-part harmonization using a set of rules to build the fitness function and musical knowledge to design the genotype and the mutation and crossover operators, but the lack of global considerations in the fitness function led to modest results. In contrast, Maddox and Otten (2000) got good results implementing a system very similar to McIntyre's (1994), but using a more flexible representation, resulting in a larger search space of possible individuals, and without the tiered structure in the fitness function, enabling a less constrained search process.

Another good example is species counterpoint: Polito et al. (1997) extracted rules for species counterpoint from a classic eighteenth century music treatise, using them to define fitness functions in a multi-agent genetic programming system: each agent performed a 


\begin{tabular}{|c|c|c|}
\hline Reference & Composition task & Comments \\
\hline \hline McIntyre, 1994 & four-part harmonization & $\begin{array}{c}\text { explores several schemes to combine } \\
\text { rules into the fitness function }\end{array}$ \\
\hline Horner \& Ayers, 1995 & four-part harmonization & $\begin{array}{c}\text { two stages: enumeration of possible } \\
\text { chord voicings, evolutionary algorithm } \\
\text { for voice-leading rules }\end{array}$ \\
\hline Phon-Amnuaisuk et al., 1999 four-part harmonization & $\begin{array}{c}\text { criticizes vanilla evolutionary algorithms } \\
\text { for generating unstructured } \\
\text { harmonizations }\end{array}$ \\
\hline Maddox \& Otten, 2000 & four-part harmonization & similar to McIntyre's (1994) \\
\hline Polito et al., 1997 & species counterpoint & multi-agent genetic programming system \\
\hline Gwee, 2002 & species counterpoint & fuzzy rules \\
\hline
\end{tabular}

Table 7: References for Section 3.2.2, in order of appearance.

set of composition or transformation operations on a given melody specified as a seed, and they cooperated to produce the composition. Gwee (2002) exhaustively studied the computational complexity of problems related to the generation of species counterpoint with rulesets, and implemented an evolutionary algorithm whose fitness function was based on a set of fuzzy rules (although he also experimented with trained artificial neural networks as fitness functions).

\subsubsection{Constraint Satisfaction}

Gradually (in a process that spanned the 1980s and 1990s), some researchers on algorithmic composition with rule-based systems adopted formal techniques based on logic programming. For example, Boenn et al. (2008) used answer set programming to encode rules for melodic composition and harmonization. However, most of the work on logic programming has been under a different paradigm: the formulation of algorithmic composition tasks as constraint satisfaction problems (CSPs). Previously referenced work, as Steels's (1979), Levitt's (1981), Schottstaedt's (1989) and Löthe's (1999) can be seen as part of a gradual trend towards the formulation of musical problems as $\operatorname{CSPs}^{17}$, although constraint logic programming (CLP) came to be the tool of choice to solve CSPs. Good surveys on CLP for algorithmic composition have been written by Pachet and Roy (2001) and Anders and Miranda (2011).

Ebcioğlu worked for many years in this area, achieving notable results. In a first work implemented in Lisp (Ebcioğlu, 1980), he translated rules of fifth-species strict counterpoint to composable Boolean functions (he had to add rules of his own to bring the system into producing acceptable results, though), and used an algorithm that produced an exhaustive enumeration of the compositions satisfying a previously arranged set of rules: basically, he implemented a custom engine for logic programming in Lisp. Over the next decade, he the tackled the problem of writing four-part chorales in the style of J. S. Bach. Finally, he produced CHORAL, a monumental expert system (Ebcioğlu, 1988), distilling into it 350 rules to guide the harmonization process and the melody generation. To keep the problem

17. While Gill's (1963) implementation was formulated as a CSP, it was somewhat primitive by later standards. 
tractable, he designed a custom logic language (BSL) with optimizations over standard logic languages, as backjumping. His system received substantial publicity, and was supposed to reach the level of a talented music student, in his own words.

Following Ebcioğlu's work, many constraint systems have been implemented for harmonization or counterpoint. Tsang and Aitken (1991) implemented a CLP system using Prolog to harmonize four-part chorales. However, their system was grossly inefficient. ${ }^{18}$ Ovans and Davison (1992) described an interactive CSP system for first-species counterpoint, where a human user drove the search process, and the system constrained the possible outputs (according to counterpoint rules) as the search progressed. They took care of efficiency by using arc-consistency in the resolution of the constraints. Ramírez and Peralta (1998) solved a different problem: given a monophonic melody, their CLP system generated a chord sequence to harmonize it. Phon-Amnuaisuk (2002) implemented a constraint system for harmonizing chorales in the style of J. S. Bach, but with an innovation over previous systems: to add knowledge to the system about how to apply the rules and control the harmonization process explicitly, thus modulating the search process in an explicit and flexible way. Anders and Miranda (2009) analyzed a Schoenberg's textbook on the theory of harmony, programming a system in Strasheela (see below) to produce self-contained harmonic progressions, instead of harmonizing pre-existing melodies, as most other constraint systems do.

While many CLP systems have been implemented to solve classical problems in harmonization or counterpoint, some researchers have studied the application of CLP techniques to different problems. In a very simple application, Wiggins (1998) used a CLP system to generate short fragments of serial music. Zimmermann (2001) described a two-stage method, where both stages used CLP: the first stage (AARON) took as input a "storyboard" to specify the mood of a composition as a function of time, and generated a harmonic progression and a sequence of directives. The second (COMPOzE) generated a four-part harmonization according to the previously arranged progression and directives; the result was intended as background music. Laurson and Kuuskankare (2000) studied constraints for the instrumentation ${ }^{19}$ of guitars and trumpets (i.e., constraints for composing music easily playable in these instruments). Chemillier and Truchet (2001) analyzed two CSPs: a style of Central African harp music, and Ligeti textures. They used heuristic search in their analyzes instead of backtracking, heralding OMClouds' approach to constraint programming (see below). Sandred (2004) proposed the application of constraint programming to rhythm.

Several general-purpose constraint programming systems for algorithmic composition have been proposed (i.e., languages and environments to program the constraints). One of the earliest examples was Courtot's (1990) CARLA, a CLP system for generating polyphonies with a visual front-end and a rich, extendable type system designed to represent relationships between different musical concepts. Pachet and Roy (1995) implemented another general-purpose musical CLP (Backtalk) in an object-oriented framework (MusES), designing a generator of four-part harmonizations on top of it. Their key contribution was a hierarchical arrangement of constraints on notes and chords, dramatically decreasing the (both cognitive and computational) complexity of the resulting constraint system.

18. In spite of using just 20 rules, it required up to 70 megabytes of memory to harmonize a phrase of 11 notes.

19. That is to say, take into account the way an instrument is played when composing its part. 


\begin{tabular}{|c|c|c|}
\hline Reference & Composition task & Comments \\
\hline Boenn et al., 2008 & melody and harmonization & answer set programming \\
\hline Ebcioğlu, 1980 & species counterpoint & implemented in LISP \\
\hline $\begin{array}{l}\text { Ebcioğlu, } 1988 \\
\text { (CHORAL) }\end{array}$ & four-part harmonization & implemented in a custom logic language (BSL) \\
\hline Tsang \& Aitken, 1991 & four-part harmonization & very inefficient \\
\hline Ovans \& Davison, 1992 & species counterpoint & interactive search \\
\hline Ramírez \& Peralta, 1998 & melody harmonization & simpler constraint solver \\
\hline Phon-Amnuaisuk, 2002 & four-part harmonization & explicit control over the search process \\
\hline Anders \& Miranda, 2009 & $\begin{array}{l}\text { Schoenberg's Theory of } \\
\text { Harmony }\end{array}$ & implemented in Strasheela \\
\hline Wiggins, 1998 & $\begin{array}{c}\text { Schoenberg's twelve-tone } \\
\text { technique }\end{array}$ & very simple demonstration \\
\hline $\begin{array}{l}\text { Zimmermann, } 2001 \\
\text { (Coppelia) }\end{array}$ & $\begin{array}{c}\text { structure, melody, } \\
\text { harmonization, rhythm }\end{array}$ & $\begin{array}{c}\text { two stages: harmonic plan (Aaron) and } \\
\text { execution (Compoze) }\end{array}$ \\
\hline $\begin{array}{c}\text { Laurson \& Kuuskankare, } \\
2000 \\
\end{array}$ & $\begin{array}{c}\text { guitar and trumpet } \\
\text { instrumentation }\end{array}$ & implemented with PWConstraints \\
\hline Chemillier \& Truchet, 2001 & $\begin{array}{l}\text { African harp and Ligeti } \\
\text { textures }\end{array}$ & implemented in OpenMusic \\
\hline Sandred, 2004 & rhythm & implemented in OpenMusic \\
\hline $\begin{array}{l}\text { Courtot, } 1990 \\
\text { (CARLA) }\end{array}$ & polyphony, general purpose & early general-purpose system \\
\hline $\begin{array}{c}\text { Pachet \& Roy, } 1995 \\
\text { (BackTalk) }\end{array}$ & four-part harmonization & implemented in MusEs \\
\hline Rueda et al., 1998 & polyphony, general purpose & $\begin{array}{c}\text { describes PWConstraints (implemented in } \\
\text { PatchWork) and Situation (implemented in } \\
\text { OpenMusic) }\end{array}$ \\
\hline Rueda et al., 2001 & general purpose & describes $\mathrm{PiCO}$ \\
\hline Olarte et al., 2009 & general purpose & describes $n t c c$ \\
\hline Allombert et al., 2006 & interactive improvisation & uses $n t c c$ \\
\hline Rueda et al., 2006 & interactive improvisation & uses $n t c c$ and Markovian models \\
\hline Pachet et al., 2011 & melody & integrates Markovian models and constraints \\
\hline Truchet et al., 2003 & general purpose & describes OMClouds \\
\hline Anders, 2007 & general purpose & describes Strasheela \\
\hline Sandred, 2010 & general purpose & $\begin{array}{l}\text { describes PWMC. } \\
\text { Implemented in PatchWork }\end{array}$ \\
\hline Carpentier \& Bresson, 2010 & orchestration & $\begin{array}{c}\text { uses multi-objective optimization } \\
\text { to discover candidate solutions. } \\
\text { Interfaces with OpenMusic and MAX }\end{array}$ \\
\hline Yilmaz \& Telatar, 2010 & harmonization & fuzzy logic \\
\hline Aguilera et al., 2010 & species counterpoint & probabilistic logic \\
\hline Geis \& Middendorf, 2008 & four-part harmonization & multi-objective Ant Colony Optimization \\
\hline $\begin{array}{c}\text { Herremans \& Sorensena, } \\
2012\end{array}$ & species counterpoint & variable neighborhood with tabu search \\
\hline Davismoon \& Eccles, 2010 & melody, rhythm & $\begin{array}{l}\text { uses simulated annealing to combine } \\
\text { constraints with Markov processes }\end{array}$ \\
\hline Martin et al., 2012 & interactive improvisation & implemented in MAX \\
\hline
\end{tabular}

Table 8: References for Section 3.2.3, in order of appearance. 
Rueda et al. (1998) reviewed two other early general-purpose systems, PWConstraints and Situation. PWConstraints was able to (relatively easily) handle problems in polyphonic composition through a subsystem (score-PMC), while Situation was more flexible and implemented more optimizations in its search procedures. PiCO (Rueda et al., 2001) was an experimental language for music composition that seamlessly integrated constraints, object-oriented programming and a calculus for concurrent processes. The idea was to use constraint programming to specify the voices in a composition, and to use the concurrent calculus to harmonize them. The authors also implemented a visual front-end to PiCO for ease of use, Cordial. In a similar way to $\mathrm{PiCO}$, ntcc was another language for constraint programming that implemented primitives for defining concurrent systems, although it was not specifically designed for algorithmic composition. ntcc has been proposed to generate rhythm patterns and as a more expressive alternative to PiCO (Olarte et al., 2009), and has mainly been used for machine improvisation: Allombert et al. (2006) used it as the improvisation stage of their two-stage system (the first stage used a temporal logic system to compose abstract temporal relationships between musical objects, while the ntcc stage generated concrete music realizations), and Rueda et al. (2006) used ntcc to implement a real-time system that learned a Markovian model (using a Factor Oracle) from musicians and concurrently applied it to generate improvisations. Not related to ntcc, Pachet et al. (2011) has also proposed a framework to combine constraint satisfaction and Markov processes.

OMClouds (Truchet et al., 2003) was another general-purpose (but purely visual) constraint system for composition, but its implementation set it apart from most other formal systems: internally, the constraints are translated to cost functions. Instead of the optimized tree search with backtracking usual in CLP, an adaptive tabu search was performed, seeking to minimize a solution with minimal cost. This avoids some problems inherent to constraint programming, such as overconstraining, but it cannot be guaranteed to completely navigate the search space. Anders (2007) implemented Strasheela, a system that was expressly designed to be highly flexible and programmable, aiming to overcome a perceived limitation of previous general-purpose systems: the difficulty to implement complex with constraints related to multiple aspects of the compositions process. Finally, another purely visual constraint system, PWMC, was proposed by Sandred (2010) to overcome perceived limitations of score-PMC. It was able to handle constraints concerning not only pitch structure as score-PMC, but also rhythm and metric structure.

It should be stressed that, while CLP has become the tool of choice to solve CSPs, other approaches are also used. Previously cited OMClouds is just one of these. Carpentier and Bresson (2010) implemented a mixed system for orchestration that worked in a curious way: the user fed the system with a target sound and a set of symbolic constraints; a multi-objective evolutionary algorithm found a set of orchestration solutions matching the target sound, and a local search algorithm filtered out the solutions not complying with the constraints. Yilmaz and Telatar (2010) implemented a system for simple constraint harmonization with fuzzy logic, while Aguilera et al. (2010) used probabilistic logic to solve first-species counterpoint. More exotic solutions have been proposed, as the use of Ant Colony Optimization with a multi-objective approach to solve the constraints of Baroque harmonization (Geis \& Middendorf, 2008), variable neighborhood with tabu search to solve soft constraints for first-species counterpoint (Herremans \& Sorensena, 2012), or simulated 


\begin{tabular}{|c|c|c|}
\hline Reference & Composition task & Comments \\
\hline \hline Pereira et al., 1997 & Baroque music & hierarchical analysis and representation \\
\hline $\begin{array}{c}\text { Ribeiro et al., 2001 } \\
\text { (MuzaCazUza) }\end{array}$ & Baroque music & generates a melody from a harmonic line \\
\hline Ramalho \& Ganascia, 1994 & jazz improvisation & uses a rule-based system for analysis \\
\hline Parikh, 2003 & jazz improvisation & also uses a rule system to analize music \\
\hline Eigenfeldt \& Pasquier, 2010 & jazz chord progressions & also uses Markovian models \\
\hline Sabater et al., 1998 & harmonization & also uses a rule system \\
\hline
\end{tabular}

Table 9: References for Section 3.2.4, in order of appearance.

annealing to combine constraints with Markov processes (Davismoon \& Eccles, 2010). Finally, Martin et al. (2012) presented an even more exotic approach: a real-time music performer that reacted to its environment. While some of the aspects of the music where controlled by Markov chains, others where expressed as a CSP. To solve this CSP in real time, a solution was calculated at random (but quickly) using binary decision diagrams.

\subsubsection{Case-Based Reasoning}

Case-based reasoning (CBR) is another formal framework for rule-based systems. In the CBR paradigm, the system has a database of cases, that can be defined as instances of a problem with their corresponding solutions. Usually, a case also contains structured knowledge about how the problem is solved in that case. When faced with a new problem, the system matches it against the case database. Unless the new problem is identical to one recorded in the case database, the system will have to select a case similar to the new problem, and adapt the corresponding solution to the new problem. If the new solution is deemed appropriate, a new case (recording the new problem along with the new solution) may be included in the database.

Several researchers have used CBR for algorithmic composition. Pereira et al. (1997) implemented a system that generated its case database from just three Baroque music pieces, which were analyzed into hierarchical structures; the cases were their nodes. The system composed just the soprano melodic line of the piece, searching for similar cases in its case database. The results were comparable to the output of a first-year student, according to music experts consulted by the authors. An intersecting set of researchers implemented a simpler CBR composing system (Ribeiro et al., 2001) that generated a melody from a harmonic line, this time with a case database generated from just six Baroque pieces. Cases were represented in a different way, tough: each case represented the rhythm, the melody and other attributes associated to a chord in a given context. To generate a new music piece, a harmonic line was specified, and the system fleshed out the music piece by matching the cases to the harmonic line.

Hybrid systems have also been proposed. Ramalho and Ganascia (1994) proposed a jazz improviser that used a rule system to analyze incoming events (for example, the ongoing sequence of chords) and a CBR engine to improvise. The case database was assembled by extracting patterns from transcriptions of jazz recordings, and consisted of descriptions of contexts and how to play in these contexts. During the improvisation, the current context 


\begin{tabular}{|c|c|c|}
\hline Reference & Composition task & Comments \\
\hline \hline $\begin{array}{c}\text { Haus \& Sametti, 1991 } \\
\text { (Scoresynth) }\end{array}$ & melody nets \\
\hline Lyon, 1995 & melody & Petri nets to encode Markov chains \\
\hline Holm, 1990 & melody, sound synthesis & inspired in CSP process algebra \\
\hline $\begin{array}{c}\text { Ross, 1995 } \\
\text { (MWSCCS) }\end{array}$ & melody & describes PiCO \\
\hline Rueda et al., 2001 & general purpose & uses ntcc \\
\hline Allombert et al. (2006) & interactive improvisation \\
\hline Rueda et al., 2006 & interactive improvisation & uses ntcc and Markovian models \\
\hline (Olarte et al., 2009) & general purpose, rhythms & describes $n t c c$ \\
\hline
\end{tabular}

Table 10: References for Section 3.2.5, in order of appearance.

was analyzed by the rule system, and those cases applying in the current context were extracted from the database and combined to determine the output of the improviser. Considering that Ramalho and Ganascia's system was too inflexible, Parikh (2003) implemented another jazz improviser, intending to use a large case database containing jazz fragments from various sources, in order to get a system with a style of its own. Eigenfeldt and Pasquier (2010) used a case-based system to generate variable-order Markov models for jazz chord progressions.

Outside the jazz domain, a hybrid system for harmonizing melodies of popular songs was implemented by Sabater et al. (1998): given a melody, the system sequentially decided the chords to harmonize it. If the CBR module failed to match a case, the system fell back to a simple heuristic rule system to select an appropriate chord. As the harmonized output was added to the case database, the CBR module gradually learned over time from the rule system.

\subsubsection{Concurrency Models}

Concurrency models can be described as formal languages to specify, model and/or reason about distributed systems. They provide primitives to precisely define the semantics of interaction and synchronization between several entities. Their main application has been modeling and designing distributed or concurrent computer systems, but they have also been used as languages to partially or fully model the composition process, because music composition can be formulated as an endeavor to carefully synchronize streams of music events produced by several interacting entities. Concerning algorithmic composition, the most used concurrency models have been Petri nets and several kinds of process algebras, also known as process calculi. Detailed descriptions of these models are beyond the scope of this survey; see for example Reisig's (1998) book on Petri nets and Baeten's (2005) survey on process algebras for more information.

Petri nets have been used as the basis for Scoresynth (Haus \& Sametti, 1991), a visual framework for algorithmic composition in which Petri nets were used to describe transformations of musical objects (sequences of notes and musical attributes), and the synchronization 
between musical objects was implicit in the structure of the net. Petri nets have also been used as an efficient and compact way to implement Markov chains (Lyon, 1995).

Process algebras were first used for algorithmic composition by Holm (1990), although his model (inspired by Hoare's algebra, CSP) was more geared towards sound synthesis than music composition. A more proper example is Ross's (1995) MWSCCS, an extension (adding concepts for music composition) of a previously existing algebra (WSCCS). Specifications for algorithmic composition written in MWSCCS were meant to resemble grammatical specifications, but with a richer expressive power. Later examples have also been cited in Section 3.2.3, as the PiCO language (Rueda et al., 2001), which integrated logical constraints and a process algebra. Also cited in that Section, ntcc is a process algebra that has been used to implement machine improvisation (Allombert et al., 2006) and to drive a Markovian model (using a Factor Oracle) for real-time machine learning and improvisation (Rueda et al., 2006). It has also been proposed to generate rhythm patterns, and as a more expressive alternative to PiCO (Olarte et al., 2009).

\subsection{Markov Chains and Related Methods}

Conceptually, a Markov chain is a simple idea: a stochastic process, transiting in discrete time steps through a finite (or at most countable) set of states, without memory: the next state depends just on the current state, not on the sequence of states that preceded it or on the time step. In their simplest incarnations, Markov chains can be represented as labeled directed graphs: nodes represent states, edges represent possible transitions, and edge weights represent the probability of transition between states. However, Markov chains are more commonly represented as probability matrices.

When Markov chains are applied to music composition, the probability matrices may be either induced from a corpus of pre-existing compositions (training), or derived by hand from music theory or by trial-and-error. The former is the most common way to use them in research, while the latter is more used in software tools for composers. An important design decision is how to map the states of the Markov chain to musical objects. The simplest (but fairly common) mapping just assigns a sequential group of notes to each state, with the choice of just one note (instead of a larger sequence) being fairly common.

It is also common to extend the consideration of the "current state": in an $n$-th order Markov chain, the next state depends on the last $n$ states, not just the last one. As a consequence, the probability matrix has $n+1$ dimensions. In algorithmic composition, Markov chains are mostly used as generative devices (generating a sequence of states), but they can also be used as analysis tools (evaluating the probability of a sequence of states). In the latter case, the term $n$-gram is also used, though strictly speaking it refers to a sequence of $N$ states.

Markov chains were a very popular method in the early years of algorithmic composition. Early examples have already been reviewed in Section 2.1; additionally, Ames (1989) also provides a good survey. However, Markov chains generated from a corpus of pre-existing compositions captured just local statistical similarities, and their limitations soon became apparent (Moorer, 1972): low- $n$ Markov chains produced strange, unmusical compositions

that wandered aimlessly, while high- $n$ ones essentially rehashed musical segments from the corpus and were also very computationally expensive to train. 


\begin{tabular}{|c|c|c|}
\hline Reference & Composition task & Comments \\
\hline Tipei, 1975 & melody & $\begin{array}{l}\text { Markov chains are part of a larger, } \\
\text { ad hoc system }\end{array}$ \\
\hline Jones, 1981 & melody & very simple introduction for composers \\
\hline Langston, 1989 & melody & dynamic weights \\
\hline North, 1991 & melody & $\begin{array}{l}\text { Markov chains are part of a larger, } \\
\text { ad hoc system }\end{array}$ \\
\hline $\begin{array}{l}\text { Ames \& Domino, } 1992 \\
\text { (Cybernetic Composer) }\end{array}$ & jazz, rock & uses Markov chains for rhythms \\
\hline Visell, 2004 & $\begin{array}{l}\text { real-time generative art } \\
\text { installation }\end{array}$ & $\begin{array}{l}\text { Liberal use of the concept of HMM. } \\
\text { Implemented in MAX }\end{array}$ \\
\hline $\begin{array}{c}\text { Zicarelli, } 1987 \\
\text { (M and Jam Factory) }\end{array}$ & interactive improvisation & commercial GUI applications \\
\hline Ariza, 2006 & & $\begin{array}{l}\text { alternative representation for transition } \\
\text { matrices. Implemented in athenaCL }\end{array}$ \\
\hline Ponsford et al., 1999 & composing sarabande pieces & $\begin{array}{l}\text { adds symbols to expose the structure } \\
\text { of the pieces }\end{array}$ \\
\hline Lyon, 1995 & melody & $\begin{array}{l}\text { implements Markov chains } \\
\text { with Petri nets }\end{array}$ \\
\hline Verbeurgt et al., 2004 & melody & $\begin{array}{l}\text { two stages: a Markovian model } \\
\text { and an artificial neural network }\end{array}$ \\
\hline $\begin{array}{l}\text { Thom, } 2000 \\
\text { (BoB) }\end{array}$ & interactive jazz improvisation & statistical machine learning \\
\hline Lo \& Lucas, 2006 & melody & $\begin{array}{l}\text { evolutionary algorithm, Markov chains } \\
\text { used in the fitness function }\end{array}$ \\
\hline Werner \& Todd, 1997 & melody & $\begin{array}{l}\text { co-evolutionary algorithm, Markov } \\
\text { chains used as evolvable fitness functions }\end{array}$ \\
\hline Thornton, 2009 & melody & $\begin{array}{l}\text { grammar-like hierarchy } \\
\text { of Markov models }\end{array}$ \\
\hline $\begin{array}{c}\text { Cruz-Alcázar and Vidal-Ruiz } \\
\text { (1998) }\end{array}$ & melody & $\begin{array}{l}\text { analysis with grammatical inference, } \\
\text { generation with Markovian models }\end{array}$ \\
\hline Gillick et al. (2009) & jazz improvisation & $\begin{array}{l}\text { analysis with grammatical inference, } \\
\text { generation with Markovian models }\end{array}$ \\
\hline Eigenfeldt \& Pasquier, 2010 & jazz chord progressions & uses case-based reasoning \\
\hline Davismoon \& Eccles, 2010 & melody, rhythm & $\begin{array}{l}\text { uses simulated annealing to combine } \\
\text { constraints with Markov processes }\end{array}$ \\
\hline Pachet et al., 2011 & melody & $\begin{array}{l}\text { integrates Markovian models and } \\
\text { constraints }\end{array}$ \\
\hline Grachten, 2001 & jazz improvisation & $\begin{array}{l}\text { integrates Markovian models } \\
\text { and constraints }\end{array}$ \\
\hline Manaris et al., 2011 & interactive melody improvisation & $\begin{array}{l}\text { Markov chains generate candidates } \\
\text { for an evolutionary algorithm }\end{array}$ \\
\hline Wooller \& Brown, 2005 & transitioning between two melodies & $\begin{array}{l}\text { alternates Markov chains } \\
\text { from the two melodies }\end{array}$ \\
\hline
\end{tabular}

Table 11: References for Section 3.3, in order of appearance.

Because of this, Markov chains came to be seen as a source of raw material, instead of a method to truly compose music in an automated way, except for some specialized tasks such 
as rhythm selection (McAlpine et al., 1999). Therefore, while research interest in Markov chains receded in subsequent years as these limitations became apparent and other methods were developed, they remained popular among composers. However, citing even a relevant subset of all the works were composers use Markov chains as part of their compositional process would inflate the reference list beyond reasonable length. A few typical examples of Markov chains used by composers (sometimes as part of a larger automatic compositional framework or software system) are the papers of Tipei (1975), Jones (1981), Langston (1989, although he used dynamically computed weights), North (1991) and Ames and Domino (1992). It should be noted that composers sometimes deconstruct formal methods to adapt them to their own purposes, as when Visell (2004) used the concept of a Hidden Markov Model (described below) to implement a manually-tuned real-time generative art system.

In addition, many software suites use Markov chains to provide musical ideas to composers, even if the probabilities are specified by hand instead of generated from a corpus. Ariza (2006) gives a compact list of software suites and experimental programs using Markov chains, while his thesis (Ariza, 2005b) provides a comprehensive view of the field. Much has been done for usability in this field, by using GUI interfaces (Zicarelli, 1987), but also developing more effective ways to encode the probability matrices, for example as compact string specifications (Ariza, 2006).

However, novel research on Markov chains for algorithmic compositions has still been carried out in several ways. For example, Ponsford et al. (1999) used a corpus of sarabande pieces (relatively simple dance music) to generate new compositions using Markov models ${ }^{20}$, but with a pre-processing stage to automatically annotate the compositions of the corpus with symbols to make explicit their structure, and a post-processing stage using a template to constrain the structure of the synthesized composition, in order to generate minimally acceptable results. Another way is the hybridization of Markov chains with other methods. For example, Lyon (1995) used Petri nets as an efficient and compact way to implement Markov chains, while Verbeurgt et al. (2004) used Markov chains ${ }^{21}$ to generate a basic pattern for the melody, which was then refined with an artificial neural network. In the BoB system (Thom, 2000), Markov chains were trained by statistical learning: from a set of jazz solos, statistical signatures were extracted for pitches, melodic intervals and contours. Then, these signatures were used to define the transition probabilities of a Markov chain whose output was sampled to generate acceptable solos. Lo and Lucas (2006) trained Markov chains with classic music pieces, but, instead of generating compositions with them, used them as fitness evaluators in an evolutionary algorithm to evolve melodies encoded as sequences of pitches. Werner and Todd (1997) also used Markov chains to evaluate simple (32-note) melodies, but with the particularity that the chains themselves were also subject to evolution, to investigate sexual evolutionary dynamics. Thornton (2009) defined a set of grammar-like rules from an existing composition, inferring a hierarchy of Markov models to use statistical patterns of the analyzed composition at multiple levels. As already mentioned in Section 3.1, Cruz-Alcázar and Vidal-Ruiz (1998) and Gillick et al. (2009) used grammatical inference with Markovian models. Regarding symbolic methods, Eigenfeldt and Pasquier (2010) used a case-based system to generate Markov processes for jazz chord

20. Their work is commonly cited in the literature as grammatical, but their methodology is thoroughly statistical.

21. Also reviewed in Section 3.4. 
progressions, (Davismoon \& Eccles, 2010) used simulated annealing to combine constraints and Markov processes, and Pachet et al. (2011) proposed a framework to combine Markovian generation of music with rules (constraints) to produce better results.

Additionally, Markov chains remained a feasible option for restricted problems (for example, real-time performances, as jazz improvisation), as their limitations were less apparent in these cases than in the generation of whole compositions. For example, Grachten (2001) developed a jazz improviser where Markov chains generated duration and pitches, and then a system of constraints refined the output, and pre-defined licks (short musical patterns) were inserted at appropriate times. Manaris et al. (2011) also implemented an improviser, using a Markov model trained with user input to generate a population of candidate melodies, feeding them into an evolutionary algorithm, whose fitness function rewarded melodies whose metrics were similar to the user input's metrics. A different (but also restricted) problem was studied by Wooller and Brown (2005): applying Markov chains to generate musical transitions (morphings) between two different pieces in a simple application of non-linear music, by stochastically alternating between two Markov chains, each one trained with one of the pieces.

\subsubsection{Related Methods}

More sophisticated Markovian models (and related statistical methods; see the survey in Conklin, 2003) have also been applied for algorithmic composition, as in Pachet's (2002) Continuator, a real-time interactive music system. The Continuator departs from common Markov chain implementations in that it uses variable-order (also known as mixed-order) Markov chains ${ }^{22}$, which are not constrained to a fixed $n$ value, and can be used to get the best of low and high- $n$ chains. Conklin and Witten (1995) implemented a sophisticated variableorder scheme ${ }^{23}$, whose main feature was the consideration in parallel of multiple viewpoints or sequences of events in the compositions (for example, pitches, durations, contours, etc.), instead of integrating them all in a unique sequence of symbols, as it was common for most implementations of Markov chains. Variable-order Markov chains have also been used as part of a larger real-time music accompaniment system (Martin et al., 2012). Other variableorder schemes used in algorithmic composition, formulated in a machine learning framework, are Prediction Suffix Trees (PSTs, Dubnov et al., 2003), more space-efficient structures like Factor Oracles ${ }^{24}$ (Assayag \& Dubnov, 2004), and Multiattribute Prediction Suffix Graphs (MPSGs, Triviño Rodríguez \& Morales-Bueno, 2001), which can be considered an extension of PSTs to consider multiple viewpoints as in Conklin and Witten's work. Sastry (2011) also used multiple viewpoints and PSTs to modelize Indian tabla compositions, though his model could also be used to generate new compositions.

Hidden Markov Models (HMMs) also are generalizations of Markov chains that have been used for algorithmic composition. A HMM is a Markov chain whose state is unobservable, but some state-dependent output is visible. Training a HMM involves not only

22. It should be noted that Kohonen's method (Kohonen et al., 1991), reviewed in Section 3.1.3, is similar (in some ways) to variable-order chains.

23. Conklin and Witten's method has also been described as grammatical, but they are included here because their emphasis in formal statistical analysis.

24. Also implemented with a concurrent constraint paradigm by Rueda et al. (2006). See Section 3.2.3 and Section 3.2.5 for more details. 


\begin{tabular}{|c|c|c|}
\hline Reference & Composition task & Comments \\
\hline $\begin{array}{l}\text { Pachet, } 2002 \\
\text { (Continuator) }\end{array}$ & interactive improvisation & variable-order \\
\hline Conklin \& Witten, 1995 & Bach chorales & multiple viewpoint systems \\
\hline Martin et al., 2012 & interactive improvisation & variable-order; implemented in MAX \\
\hline Dubnov et al., 2003 & melody & $\begin{array}{l}\text { Prediction Suffix Trees. } \\
\text { Implemented in OpenMusic }\end{array}$ \\
\hline Rueda et al., 2006 & interactive improvisation & uses $n t c c$ and Factor Oracles \\
\hline Assayag \& Dubnov, 2004 & melody & $\begin{array}{c}\text { Factor Oracles. } \\
\text { Implemented in OpenMusic }\end{array}$ \\
\hline $\begin{array}{l}\text { Triviño Rodríguez \& } \\
\text { Morales-Bueno, } 2001\end{array}$ & melody & Multiattribute Prediction Suffix Graphs \\
\hline Sastry, 2011 & improvisation of tabla rhythms & $\begin{array}{c}\text { multiple viewpoints and Prediction } \\
\text { Suffix Trees. } \\
\text { Implemented in MAX }\end{array}$ \\
\hline Farbood \& Schoner, 2001 & species counterpoint & Hidden Markov Models \\
\hline Biyikoglu, 2003 & four-part harmonization & Hidden Markov Models \\
\hline Allan, 2002 & four-part harmonization & Hidden Markov Models \\
\hline $\begin{array}{l}\text { Morris et al., } 2008 \\
\quad \text { (SongSmith) }\end{array}$ & melody harmonization & Hidden Markov Models \\
\hline $\begin{array}{l}\text { Schulze, } 2009 \\
\text { (SuperWillow) }\end{array}$ & $\begin{array}{l}\text { melody, rhythm, two-voice } \\
\text { harmonization }\end{array}$ & $\begin{array}{l}\text { Hidden Markov Models } \\
\text { and Prediction Suffix Trees }\end{array}$ \\
\hline Yi \& Goldsmith, 2007 & four-part harmonization & Markov Decision Processes \\
\hline Martin et al., 2010 & interactive improvisation & $\begin{array}{c}\text { Partially Observable } \\
\text { Markov Decision Processes }\end{array}$ \\
\hline
\end{tabular}

Table 12: References for Section 3.3.1, in order of appearance.

determining a matrix of transition probabilities, but also a matrix of output probabilities (that is, for each state, the probability of each possible output). Then, given a sequence of outputs, it is possible to compute the most likely sequence of states to produce that sequence of outputs, using the Viterbi dynamic programming algorithm. In this way, HMMs find a globally optimized sequence of states, while simpler Markov methods perform just local optimization. When applied to algorithmic composition, HMMs are appropriate to add elements to an existing composition (most commonly, counterpoint and harmonization), given a set of pre-existing examples: the composition is modeled as a sequence of outputs of the HMM, and the additions are computed as the most likely sequence of states of the HMM.

Farbood and Schoner (2001) implemented the earliest example of a HMM for algorithmic composition: they trained a second-order HMM to generate Palestrina-style first-species counterpoint (the simplest way to write counterpoint), defining the training set from rules used to teach counterpoint. A related problem is to train HMMs with a set of chorale harmonizations in the style of J.S. Bach in order to get more Bach-like harmonizations. This problem has been researched by Biyikoglu (2003) and Allan (2002); the latter divided the problem of harmonization into the same three subtasks as in HARMONET (Hild et al., 1992). For Microsoft's SongSmith software, Morris et al. (2008) trained a HMM with 
300 lead sheets (specifications for song melodies) to generate chords to accompany a userspecified vocal melody, parametrizing the resulting system with a very intuitive interface for non-technical users. Schulze (2009) generated music in several styles using mixed-order Markov chains to generate the melodies, and HMMs to harmonize them.

Markov Decision Processes (MDPs) are another generalization of Markov models, in which an agent maximizes some utility function by taking actions to probabilistically influence the next state, and Partially Observable MDPs (POMDPs) represent the corresponding generalization of HMMs. Experimental systems for algorithmic composition have been implemented with MDPs (Yi \& Goldsmith, 2007) and POMDPs (Martin et al., 2010), though it is not clear that these sophisticated models offer definitive advantages over simpler ones.

\subsection{Artificial Neural Networks and Related Methods}

Artificial Neural Networks (ANNs) are computational models inspired in biological neural networks, consisting of interconnected sets of artificial neurons: very simple computational devices that aggregate numeric inputs into a single numeric output using a (generally) simple but nonlinear function. Some neurons have connections that are set externally (input connections), while other have output signals intended to be read as the result of the network's computation (output connections). Typically, neurons are organized in recurrent networks (some or all neurons have inputs that come from other neurons) with several interconnected layers, and many variations can be found in the literature. ANNs are typically used as a machine learning method, using a set of examples (input patterns) to train the network (i.e., to set the weights of the connections between neurons), in order to use it to recognize or generate similar patterns. Effectively, this means that neural networks need a pre-existing corpus of music compositions (all of them in a very similar style, generally); therefore they can at most imitate the style of the training examples. Most papers use a supervised learning approach, meaning that the examples in the training set are associated with a signal, and the ANN learns this association. An important aspect of ANN design is the modelization of musical composition, that is, the mapping between music or music notation and the inputs and outputs of the network. Another important aspect is the way in which compositions are fed to the ANNs: they may be presented as temporal patterns in the network inputs, which are usually windowed in segments, but in some cases they are fed at once (as wholes) to the ANNs (these implementations do not scale well, though, because of the big ANNs needed to model long compositions).

ANNs were first used during the 1970s and 1980s to analyze musical compositions, creating artificial models of cognitive theories of music (Todd \& Loy, 1991), but they were later adapted for music composition. The first example was implemented by Todd (1989), who used a three-layered recurrent ANN designed to produce a temporal sequence of outputs encoding a monophonic melody, each output signal of the network representing an absolute

pitch. Given a set of one or more composition examples, the ANN was trained to associate a single input configuration to the output temporal sequence of the corresponding composition. Then, feeding input configurations different to the ones used during the training created melodies interpolated between the ones used during the training. If just one melody was used during the training, the result was an extrapolation from it. Later that year, Duff (1989) published another early example, but using a different approach, encoding relative 


\begin{tabular}{|c|c|c|}
\hline Reference & Composition task & Comments \\
\hline Todd, 1989 & melody & three layers, recurrent \\
\hline Duff, 1989 & melody & two layers, recurrent \\
\hline $\begin{array}{l}\text { Mozer, } 1991 \\
\text { (CONCERT) }\end{array}$ & melody & $\begin{array}{l}\text { psychologically-grounded representation } \\
\text { of pitch }\end{array}$ \\
\hline Lewis, 1991 & & $\begin{array}{l}\text { feedforward model, used as the fitness } \\
\text { function in an optimization algorithm }\end{array}$ \\
\hline Shibata, 1991 & harmonization & feedforward model \\
\hline Bellgard \& Tsang, 1992 & harmonization & effective Boltzmann machine \\
\hline Melo, 1998 & harmonization & $\begin{array}{l}\text { the ANN is trained } \\
\text { to model music tension }\end{array}$ \\
\hline Toiviainen, 1995 & jazz improvisation & recurrent model \\
\hline Nishijimi \& Watanabe, 1993 & jazz improvisation & feedforward model \\
\hline Franklin, 2001 & jazz improvisation & recurrent model \\
\hline $\begin{array}{l}\text { Hild et al., } 1992 \\
\text { (HARMONET) }\end{array}$ & four-part harmonization & $\begin{array}{c}\text { three-layered architecture } \\
\text { (two ANNs and a constraint system) }\end{array}$ \\
\hline $\begin{array}{l}\text { Feulner \& Hörnel, } 1994 \\
\text { (MELONET) }\end{array}$ & four-part harmonization & $\begin{array}{l}\text { uses HARMONET and another ANN } \\
\text { for melodic variations }\end{array}$ \\
\hline $\begin{array}{l}\text { Goldman et al., } 1996 \\
\text { (NETNEG) }\end{array}$ & species counterpoint & $\begin{array}{l}\text { ANN for basic melody, an ensemble of } \\
\text { agents refine the melody }\end{array}$ \\
\hline Verbeurgt et al., 2004 & melody & $\begin{array}{l}\text { two stages: a Markovian model } \\
\text { and an ANN }\end{array}$ \\
\hline Adiloglu \& Alpaslan, 2007 & species counterpoint & feedforward model \\
\hline Browne \& Fox, 2009 & melody & $\begin{array}{l}\text { simulated annealing with an ANN } \\
\text { to measure musical tension }\end{array}$ \\
\hline Coca et al., 2011 & melody & $\begin{array}{l}\text { recurrent model, uses chaotic non-linear } \\
\text { systems to introduce variation }\end{array}$ \\
\hline
\end{tabular}

Table 13: References for Section 3.4, in order of appearance.

instead of absolute pitches (as Todd's work) in the mapping, for composing music in Bach's style.

As a machine learning paradigm, ANNs can be used in many different ways, so Todd's approach is not the only possible; indeed, other early papers provide different examples. For example, Mozer (1991) developed a recurrent ANN with a training program devised to capture both local and global patterns in the set of training examples. The model also featured in the output mapping a sophisticated multidimensional space for pitch representation, to capture a formal psychological notion of similarity between different pitches. In this way, similar output signals are mapped to similar pitches, in order to facilitate the learning phase and improve the composition phase. Lewis (1991) proposed another ANN framework: creation by refinement, in which a feedforward ANN was trained with a set of patterns ranging from random to very good music, associating each pattern with a (possibly) multidimensional musicality score. In this way, the training phase generated a mapping function from patterns to musicality scores. Then, to create new compositions, the mapping was inverted: starting from a purely random pattern, a gradient-descent algorithm used the ANN as a 
critique, reshaping the random pattern to maximize the musicality score in the hope of finally producing a pleasant composition. Unfortunately, this paradigm had a prohibitive computational cost, so it was tested only with fairly simple and short compositions.

Most of the early examples described above were experiments at composing more or less full-fledged monophonic compositions. However, ANNs were also used to automate other tasks in music composition, as harmonization of pre-existing melodies. Shibata (1991) implemented an early example: a feedforward ANN that represented chords using their component tones, trained for harmonizing simple MIDI music, whose performance was measured by human listeners. A more sophisticated ANN used for harmonization, the effective Boltzmann machine (EBM), also provided a measure of the quality of the output relative to the training set (Bellgard \& Tsang, 1992). Melo (1998) also harmonized classical music, but with a notable twist: in order to model the tension ${ }^{25}$ in the music to be harmonized, he measured the tension curve reported by several human subjects while listening to the music, and then used an averaged tension curve to train an ANN, such that the chord progressions generated by the ANN matched the tension level suggested by the curve. As it can be seen, harmonization was a popular test case, but other problems were also tried. For example, Toiviainen (1995) used ANNs to generated jazz improvisations based on a set of training examples. The ANNs were able to create new jazz melodic patterns based on the training set. In a similar way, Nishijimi and Watanabe (1993) trained a set of feedforward ANNs to produce jazz improvisations in a jam session, by modeling several music features of jazz and using examples of modeled jazz improvisations to train the ANNs. Franklin (2001) used a recurrent ANNs to improvise jazz (trade four solos with a jazz performer), trained in two phases: a first phase training an ANN with a set of pre-specified examples, and a second phase where the ANN is reconfigured and trained by reinforcement learning, where the reinforcement values are obtained by applying a set of heuristic rules.

Some researchers came to use hybrid systems, combining ANNs with other methods. One of the first examples was HARMONET (Hild et al., 1992): a model designed to solve a more complex task: four-part choral harmonization in Bach's style. HARMONET had a three-layered architecture: the first component was a feedforward ANN with a sophisticated encoding of musical information (optimized for harmonization functions instead of individual pitches), which was used to extract harmonization information. The output was fed to the second component, a rule-based constraint satisfaction algorithm to generate the chords, and the final component was another ANN designed to add quaver ornaments to the previously generated chords. As an evolution of HARMONET, MELONET (Feulner \& Hörnel, 1994; improved by Hörnel \& Degenhardt, 1997) not only harmonized chorales, but also generated melodic variations for their voices, using HARMONET as a first processing stage for the harmonization, then used another neural network to generate the melodic variations.

NETNEG (Goldman et al., 1996) was another hybrid system that used an ANN trained with sixteenth century classical music compositions. The ANN generated a basic melody by segments. After each segment was created, an ensemble of agents generated a polyphonic elaboration of the segment. The agents had rule-based systems crafted from music theoret-

25. An important property of music, rather difficult to define. At each point in time, the tension is related to the interplay between structure and uncertainty perceived by the listener in the flow of the music. Informally, it can be defined as the unfinishedness of the music if it were stopped at that point. 
ical considerations, and coordinated to maintain a coherent global output. ANNs can also be combined with probabilistic methods: in the work of Verbeurgt et al. (2004), a set of training sequences was decomposed into musical motifs, encoded in relative pitch. Then, a Markov chain was constructed, whose states were the motifs. New compositions were generated by the Markov chain, but to assign the absolute pitches to the motifs in the resulting composition, they trained an ANN. Adiloglu and Alpaslan (2007) used feedforward ANNs to generate two-voice counterpoint, applying notions of music theory to the representation of musical information in the networks. In Browne and Fox's (2009) system, simulated annealing was used to arrange small fragments (motifs) of classical music, trying to get a profile of musical tension (the same metric as in Melo, 1998) similar to the profile of a pre-specified composition, measured using an ANN specialized in music perception. Finally, another hybrid system was implemented by Coca et al. (2011), which used ANNs trained with pre-existing compositions together with pseudo-random musical input generated from a chaotic system, in order to generate more complex compositions in the synthesis phase.

\subsubsection{ANNs With Evolutionary Algorithms}

Among hybrid systems, those combining ANNs with evolutionary algorithms quickly became the most popular. Usually, an ANN was trained to act as the fitness function of an evolutionary algorithm. This is the case of the earliest example of these hybrid systems, NEUROGEN (Gibson \& Byrne, 1991). Its fitness function was the composed result of two ANNs, one for judging the intervals between pitches and the other for the overall structure. A genetic algorithm with a rather rigid, classical binary representation was used, severely limiting the applicability of the whole implementation. However, there are also "inverted" frameworks where the evolving individuals are ANNs. For example, Hörnel and Ragg (1996) evolved HARMONET networks, but the fitness was the network performance in training and harmonization. In another example (Chen \& Miikkulainen, 2001), recurrent three-layered ANNs evolved to compose music, and the fitness was computed from a set of rules from music theory.

Given the modular nature of evolutionary algorithms and the perceived complexity of ANNs, it is not uncommon that the evolutionary framework is laid down in a first research work, and only in subsequent developments ANNs are used to replace the original fitness function. ${ }^{26}$ For example, Spector and Alpern (1994) developed a genetic programming (GP) framework for jazz improvisations: the individuals were programs composed by collections of transformations that produced improvisations upon being fed previously existing jazz melodies, and the fitness function aggregated several simple principles from jazz music theory. The following year (Spector \& Alpern, 1995), they updated their model to train an ANN to be used as their fitness function. However, this scheme does not always fare well, specially if the initial framework uses interactive fitness. This is the case of GenJam (Biles, 1994), an evolutionary algorithm for generating jazz melodies with an interactive fitness function. Later on (Biles et al., 1996), as the interactive fitness evaluation represented a severe fitness bottleneck, ANNs were tried to partially offload evaluation from human users, with no success, as the ANNs failed to satisfactorily generalize the evaluations from their

26. This approach is risky, though, because evolutionary algorithms tend to find and exploit unexpected and undesired quirks in any fitness evaluation function; more so if the evaluator is an ANN. 


\begin{tabular}{|c|c|c|}
\hline Reference & Composition task & Comments \\
\hline Gibson \& Byrne, 1991 & melody, rhythm & Two ANNs to define the fitness function \\
\hline Hörnel \& Ragg, 1996 & melody harmonization & $\begin{array}{l}\text { evolves HARMONET, the fitness is the ANN's } \\
\text { performance in training and harmonization }\end{array}$ \\
\hline Chen \& Miikkulainen, 2001 & melody & $\begin{array}{l}\text { evolves recurrent networks, } \\
\text { the fitness is computed from a set of rules }\end{array}$ \\
\hline Spector \& Alpern, 1994 & melody & genetic programming, ANNs as fitness functions \\
\hline Biles et al., 1996 & jazz improvisation & ANNs as fitness functions \\
\hline $\begin{array}{l}\text { Johanson \& Poli, } 1998 \\
\text { (GP-Music) }\end{array}$ & melody & genetic programming, ANNs as fitness functions \\
\hline Klinger \& Rudolph, 2006 & melody & ANNs and decision trees as fitness functions \\
\hline Manaris et al., 2007 & melody & genetic programming, ANNs as fitness functions \\
\hline Burton, 1998 & drum rhythms & $\begin{array}{l}\text { Adaptive Resonance Theory } \\
\text { (unsupervised learning) as fitness function }\end{array}$ \\
\hline Phon-Amnuaisuk et al., 2007 & melody & $\begin{array}{l}\text { genetic programming, self-organinzing map } \\
\text { (unsupervised learning) as fitness function }\end{array}$ \\
\hline
\end{tabular}

Table 14: References for Section 3.4.1, in order of appearance.

training sets. In the case of the GP-Music System (Johanson \& Poli, 1998), which used GP with procedural representations of short melodies, the trained ANNs were not a failure, but decidedly below par with respect to the performance of the algorithm with interactive fitness. Klinger and Rudolph (2006) compared the performance of feedforward ANNs with learned decision trees, finding that the latter performed better and their ratings were easier to understand. In spite of these examples, more successful instances do exist: Manaris et al. (2007) extracted several statistical metrics from music compositions and trained an ANN to recognize compositions whose metrics' distributions featured Zipf's law, then used it as the fitness function in a GP framework whose individuals were procedural representations of polyphonic compositions. The results were validated as aesthetically pleasing by human testers.

All the research described up to this point uses ANNs with supervised learning. However, methods using unsupervised learning also exist. Burton (1998) proposed a genetic algorithm with a classical binary representation for generating multi-voice percussion rhythms, whose fitness function presented an unconventional feature mix. It used Adaptive Resonance Theory (ART), an ANN with unsupervised learning, initially trained to perform automatic clustering of a set of drum rhythms. Then, during the execution of the genetic algorithm, the unsupervised learning continued. The fitness was a measure of how near was the individual to some cluster. If the individual represented a brand new rhythm so different as to add a new cluster, the rhythm was presented to the user to decide if it was musically acceptable. In this way, Burton tried to get the best of interactive and automatic fitness evaluation. A different example with unsupervised learning was presented by Phon-Amnuaisuk et al. (2007), able to generate variations over a pre-specified composition. It used GP whose individuals were procedural representations of melodies, while the fitness was the similarity to the pre-specified composition, as measured by a self-organizing map (SOM) previously trained with musical elements of the pre-specified composition. 


\begin{tabular}{|c|c|c|}
\hline Reference & Composition task & Comments \\
\hline \hline Baggi, 1991 & jazz improvisation & ad hoc connectionist expert system \\
\hline Laine, 2000 & simple rhytms & uses central pattern generators \\
\hline Dorin, 2000 & poly-rhythmic musical patterns & uses Boolean networks \\
\hline Hoover et al., 2012 & acompaniment & uses CPPNs \\
\hline
\end{tabular}

Table 15: References for Section 3.4.2, in order of appearance.

\subsubsection{Related Methods}

Finally, this subsection presents methods that may be considered roughly similar to ANNs, or more generally, connectionist. For example, Neurswing (Baggi, 1991) may be described as an ad hoc expert system for jazz improvisation crafted in a connectionist framework. Surprisingly, many research papers cite Neurswing as an ANN framework, despite Baggi's disclaimer: "[Neurswing], though only vaguely resembling a neural net or a connectionist system, [...]" (Baggi, 1991). Laine (2000) used very simple ANNs to implement Central Pattern Generators, whose output patterns where then interpreted as more or less simple rhythms (motion patterns in his terminology).

Boolean networks are another connectionist paradigm in which each node has a binary state and the edges between nodes are directed; each node's state changes in discrete steps according to a (usually randomly chosen) Boolean function whose inputs are the states of the nodes with connections to that node. They may be considered as generalizations of cellular automata, and depending on the wiring and the distribution of Boolean functions, their states can change in very complex patterns, with potentially complex responses to external forcing. Because of these properties, Dorin (2000) used Boolean networks to generate complex poly-rhythmic musical patterns, modulable in real time by the user.

Hoover et al. (2012) proposed another connectionist approach: compositional pattern producing networks (CPPNs). These are feedforward networks where each "neuron" may use a different, arbitrary function, instead of the classical sigmoid function. They are usually designed by interactive evolutionary methods, and can be used to generate or modulate highly complex patterns. In the cited paper, they were fed a pre-existing simple composition as input, in order to generate an accompaniment for it.

\subsection{Evolutionary and Other Population-Based Methods}

Most evolutionary algorithms (EAs) approximately follow a common pattern: a changing set of candidate solutions (a population of individuals) undergoes a repeated cycle of evaluation, selection and reproduction with variation. The first step is to generate the candidate solutions of the initial set, either from user-specified examples or in a more or less random way. Each candidate is then evaluated using a fitness function, a heuristic rule to measure its quality. The next phase is selection: a new set of candidate solutions is generated by copying candidate solutions from the old one; each candidate solution is copied a number of times probabilistically proportional to its fitness. This step decreases the diversity of the population, which is restored by applying (to a fraction of the candidate solutions) some operators designed to increase the variation (for example, mutation or recombination 
operators). These steps are applied iteratively; as a result, best and mean fitness gradually tend to increase.

While this algorithmic pattern is common to all EAs, there exist many different algorithms using different sets of selection rules, variation operators and solution encoding. In an EA, the encoded form of a candidate solution is the genotype, while the phenotype is the translation of that coded form into a solution. Holland's original formulation of genetic algorithms is strongly associated with a plain and direct encoding of genotypes as binary strings, but this is not the case in most papers using EAs. Because of this, the term evolutionary is preferred over genetic in this paper. Another popular variant, Koza's genetic programming, represents genotypes as tree structures, often encoding expressions of a programming language, while the phenotype is the result of evaluating these expressions.

Since EAs are particularly prone to be hybridized with other methods, they are also reviewed in the other sections: with grammars in Section 3.1.2, with ANNs in Section 3.4.1, with Markov chains in Section $3.3^{\dagger}$, with rule-based systems in Section 3.2.2, and with cellular automata in Section 3.6.1 ${ }^{\ddagger}$. In general, the papers cited in these sections will not be discussed here. We also recommend some literature to learn more about evolutionary computer music: Burton and Vladimirova (1999) wrote a survey with long, thorough descriptions of the referenced papers, while the survey of Santos et al. (2000) is more similar to ours in style, packed with brief descriptions. Miranda and Biles's (2007) book is more recent, but also contains work on other optimization methods (as swarm optimization) and algorithmic composition techniques (as cellular automata).

\subsubsection{Evolution with Automatic Fitness Functions}

The difficulty to define automatic fitness functions has been a constant issue, frequently limiting the application of evolutionary methods to well-defined and restricted problems in composition. Horner and Goldberg (1991) provided one of the first examples: they implemented an EA for thematic bridging, a composition technique consisting of defining a sequence (with a pre-specified preferred length) of small musical patterns such that the first and the last ones are pre-specified, and each pattern is the result of applying a simple transformation to the previous one. Naturally, the individuals in the EA were defined as lists of operations applied to the initial pattern to generate the sequence of patterns. The fitness measured how close the final pattern (generated from the operations) was to the pre-specified final pattern, plus the difference between actual and preferred lengths of the sequence. Essentially the same work (with differences in the underlying representation and operation set) was reported by Ricanek et al. (1993).

A common way to implement a fitness function is as a weighted sum of features of the composition (although tuning the weights to optimize the EA can prove difficult except for toy problems). For example, Marques et al. (2000) composed short polyphonic melodies using a very direct representation for the genotypes and also a simple fitness function, with a rather simple, ad hoc evaluation of harmony and melodic value. The results were reportedly acceptable. Johnson et al. (2004) also composed short melodies using an EA

$\dagger$. Only a few examples of evolutionary algorithms were described in this section, not warranting a dedicated subsection for them.

$\ddagger$. Same case as in the previous note. 


\begin{tabular}{|c|c|c|}
\hline Reference & Composition task & Comments \\
\hline Horner \& Goldberg, 1991 & thematic bridging & fitness: distance to original melodies \\
\hline Ricanek et al., 1993 & thematic bridging & fitness: distance to original melodies \\
\hline Marques et al., 2000 & polyphony & fitness: combination of features \\
\hline Johnson et al., 2004 & melody & fitness: combination of features \\
\hline $\begin{array}{c}\text { Papadopoulos \& Wiggins, } \\
1998\end{array}$ & jazz improvisation & fitness: combination of features \\
\hline $\begin{array}{l}\text { Harris, } 2008 \\
\text { (JazzGen) }\end{array}$ & jazz improvisator & fitness: combination of features \\
\hline Towsey et al., 2001 & melodic extension & $\begin{array}{c}\text { fitness: combination of features } \\
\text { (only fitness, no evolutionary algorithm) }\end{array}$ \\
\hline Birchfield, 2003 & melody, rhythm & fitness: combination of features \\
\hline Garay Acevedo, 2004 & species counterpoint & fitness: combination of features \\
\hline Lozano et al., 2009 & $\begin{array}{c}\text { melody } \\
\text { harmonization }\end{array}$ & fitness: combination of features \\
\hline De Prisco et al., 2010 & unfigured bass & multi-objective optimization \\
\hline Freitas \& Guimarães, 2011 & $\begin{array}{c}\text { melody } \\
\text { harmonization }\end{array}$ & multi-objective optimization \\
\hline Gartland-Jones, 2002 & $\begin{array}{l}\text { generate variations } \\
\text { on two melodies }\end{array}$ & fitness: distance to a melody \\
\hline Alfonseca et al., 2005 & melody & fitness: distance to corpus of melodies \\
\hline $\begin{array}{l}\text { Özcan \& Erçal, } 2008 \\
\text { (AMUSE) }\end{array}$ & $\begin{array}{l}\text { generate variations } \\
\text { on a melody }\end{array}$ & fitness: combination of features \\
\hline Wolkowicz et al., 2009 & melody & fitness: distance to corpus of melodies \\
\hline Laine \& Kuuskankare, 1994 & melody & fitness: distance to a melody. Genetic programming \\
\hline Spector \& Alpern, 1994 & jazz improvisation & fitness: combination of features. Genetic programming \\
\hline Dahlstedt, 2007 & $\begin{array}{l}\text { contemporary } \\
\text { classical music }\end{array}$ & fitness: combination of features. Genetic programming \\
\hline Espí et al., 2007 & melody & $\begin{array}{l}\text { fitness: combination of features extracted fropm a } \\
\text { corpus of melodies. Genetic programming }\end{array}$ \\
\hline Jensen, 2011 & melody & $\begin{array}{l}\text { fitness: distance to a corpus of melodies. } \\
\text { Genetic programming }\end{array}$ \\
\hline $\begin{array}{c}\text { Díaz-Jerez, 2011; } \\
\text { Sánchez-Quintana et al., } \\
2013\end{array}$ & $\begin{array}{c}\text { contemporary } \\
\text { classical music, other } \\
\text { genres }\end{array}$ & sophisticated indirect encoding \\
\hline
\end{tabular}

Table 16: References for Section 3.5.1, in order of appearance.

with a fitness function that was a weighted sum of a series of very basic, local features of the melody. Papadopoulos and Wiggins (1998) implemented a system that, given a chord progression, evolved jazz melodies by relative pitch encoding, using as fitness function a weighted sum of eight evaluations of characteristics of the melody, ranging from very simple heuristics about the speed and the position of the notes to user-specified contour and similarity to user-specified music fragments. A similar approach was implemented by Harris (2008), with modest (if promising) results. While Towsey et al. (2001) did not actually implement an EA, they discussed how to build a fitness function for melodic extension 
(given a composition, extend it for a few bars): they proposed to extract 21 statistical characteristics from a corpus of pre-specified compositions, defining the fitness function as a weighted sum of the distance between the individual and the mean of the characteristic. In a similar vein, Birchfield (2003) implemented a fitness function as a giant weighted sum of many features in a hierarchical EA, with multiple population levels, each individual in a population being composed of individuals from lower populations (similar to the model described in Biles, 1994; see Section 3.5.2). He used the output of the EA as material to arrange a long composition for ten instruments. Garay Acevedo (2004) implemented a simple EA to compose first species counterpoint, but the features weighted in the fitness function were too simplistic, leading to modest results. Lozano et al. (2009) generated chord sequences to harmonize a pre-specified melody in two steps: first a simple EA generated a set of possible solutions according to simple local considerations (appropriateness of each chord for the corresponding part of the melody) between the chords and the notes of the melody, and then a variable neighborhood search was used to establish a chord progression according to global considerations.

The alternative to implementing the fitness function as a weighted sum of musical features is to use multi-objective evolutionary algorithms (MOEAs). However, these have very rarely been used, most probably because they are harder to implement, both conceptually and in practice. MOEAs have been used by De Prisco et al. (2010) to harmonize the unfigured bass ${ }^{27}$ and by Freitas and Guimarães (2011) for harmonization.

Another approach consists of measuring the fitness as the distance to a target composition or corpus of compositions. For example, Gartland-Jones (2002) implemented an EA to compose hybrids between two pre-specified compositions (a goal similar to Hamanaka et al.'s, 2008, cited in Section 3.1) by using one to seed the initial population, and the distance to the other (sum of difference in pitch for each note) as the fitness function. Alfonseca et al. (2005) used a more sophisticated evaluation: the fitness of each composition in the population was the sum of distances to a corpus of pre-specified target compositions. The metric was the normalized compression distance, a measure of how different two symbol strings are, based on their compressed lengths (both concatenated and separated). Özcan and Erçal (2008) used a simple genetic representation and a fitness function based on a weighted sum of a long list of simple musical characteristics, reportedly being able to generate improvisations over a pre-specified melody with a given harmonic context, but they did not specify how the evolved melodies were related to the pre-specified music. In the EA implemented by Wolkowicz et al. (2009), individuals were encoded using relative pitches, and a sophisticated statistical analysis of $n$-gram sequences in a pre-specified corpus of compositions was used to implement the fitness function.

Genetic programming has also been used with fitness functions based on comparisons with pre-specified music. In the work of Laine and Kuuskankare (1994), the individuals were discrete functions of time whose output (phenotype) was interpreted as a sequence of pitches. The fitness was simply the sum of differences in pitches between a pre-specified target composition and the phenotype of each individual, in a similar way to GartlandJones (2002). However, it is frequent to find fitness functions based on the analysis of characteristics of the compositions evolving in the algorithm, often comparing them against

27. For a description of the unfigured bass, see the discussion of Rothgeb's (1968) work in Section 3.2. 
characteristics of a pre-specified training set of compositions. Spector and Alpern (1994) used genetic programming for jazz improvisation, "trading fours": the individuals were functions that took as input a "four" and produced another one by applying a series of transformations to it. The fitness was determined by applying a series of score functions that measured the rhythm, tonality and other features of the produced "four", comparing them against a database of high-quality examples from renowned artists.

Also using genetic programming, Dahlstedt (2007) composed relatively short contemporary classical music pieces, with a simple fitness function: a set of target values were assigned for several statistics of the compositions (as note density or pitch standard deviation, among others), and the fitness was a weighted sum of the differences between the target values and the values for each individual. The individuals were trees whose nodes represented notes and different operations over musical sequences (using a developmental process from genotype to phenotype). Reportedly, the generated pieces were of acceptable quality, because the genotype model was especially well suited for contemporary classical music. Espí et al. (2007) used a simple tree representation for compositions (but without indirect encoding), defining the fitness function as a weighted sum of sophisticated statistical models for melody description (measuring the distance to the values of a set of pre-specified compositions) and several relatively simple characteristics of the composition. Jensen (2011) also used a simple tree representation for compositions; the fitness was calculated measuring frequency distributions of simple events in the compositions, rating them according to Zipf's law and similarity to pre-specified compositions.

One of the latest and most successful results in evolutionary computer music follows an evo-devo strategy. Iamus is a computer cluster which hybridizes bioinspired techniques: compositions evolve in an environment ruled by formal constraints and aesthetic principles (Díaz-Jerez, 2011). But compositions also develop from genomic encodings in a way that resembles embryological development (hence the evo-devo), providing high structural complexity at a relatively low computational cost. Each composition is the result of an evolutionary process where only the instruments involved and a preferred duration have been specified, and are included in the fitness function. Iamus can write professional scores of contemporary classical music, and it has published its debut album in September 2012 (Ball, 2012; Coghlan, 2012), with ten works interpreted by first-class musicians (including the LSO for the orchestra piece). Melomics, the technology behind this avant-garde computer-composer, is also mastering other genres and transferring the result to industry (Sánchez-Quintana et al., 2013). After compiling a myriad of musical fragments of most essential styles in a browsable, web-based repository (Stieler, 2012). For the first time, Melomics is offering music as a real commodity (priced by size of its MIDI representation), where ownership over a piece is directly transferred to the buyer.

\subsubsection{Musical IGAS}

From the previous exposition, it is apparent that designing an objective and convenient fitness function for evaluating music compositions is a very difficult problem. ${ }^{28}$ If the music is to be evaluated in terms of subjective aesthetic quality, it may become impractical or

28. To the point that artists sometimes find "unconventional" ways around this problem: Waschka (1999) argued to have solved the problem by assigning a purely random fitness value to the individuals. 


\begin{tabular}{|c|c|c|}
\hline Reference & Composition task & Comments \\
\hline Hartmann, 1990 & melody & inspired by Dawkins' biomorphs \\
\hline Nelson, 1993 & melody & inspired by Dawkins' biomorphs \\
\hline Horowitz, 1994 & rhythms & inspired by Dawkins' biomorphs \\
\hline Pazos et al., 1999 & rhythms & binary genotype \\
\hline Degazio, 1996 & melody & binary genotype; graphical representation \\
\hline $\begin{array}{l}\text { Biles, } 1994 \\
\text { (GenJam) }\end{array}$ & jazz improvisation & $\begin{array}{l}\text { two hierarchically structured populations } \\
\text { (measures and jazz phrases) }\end{array}$ \\
\hline Tokui \& Iba, 2000 & rhythms & $\begin{array}{l}\text { two hierarchically structured populations } \\
\text { (short and long rhythmic patterns). } \\
\text { Genetic programming }\end{array}$ \\
\hline Jacob, 1995 & melody & the user trains "critics" that act as fitness functions \\
\hline Schmidl, 2008 & melody & the user trains "critics" that act as fitness functions \\
\hline Putnam, 1994 & melody & genetic programming \\
\hline Ando \& Iba, 2007 & melody & genetic programming \\
\hline $\begin{array}{l}\text { MacCallum et al., } 2012 \\
\quad \text { (DarwinTunes) }\end{array}$ & melody & genetic programming \\
\hline $\begin{array}{l}\text { Kaliakatsos-Papakostas } \\
\text { et al., } 2012\end{array}$ & $\begin{array}{l}\text { melody, 8-bit sound } \\
\text { synthesis }\end{array}$ & genetic programming \\
\hline Hoover et al., 2012 & acompaniment & uses CPPNs \\
\hline McDermott \& O'Reilly, 2011 & $\begin{array}{l}\text { interactive generative } \\
\text { music }\end{array}$ & similar to CPPNs \\
\hline Ralley, 1995 & melody & minimizes user input by clustering candidates \\
\hline Unehara \& Onisawa, 2001 & melody & minimizes user input with elitism \\
\hline Díaz-Jerez, 2011 & $\begin{array}{l}\text { contemporary classical } \\
\text { music }\end{array}$ & $\begin{array}{l}\text { minimizes user fatigue producing small, good } \\
\text { compositions }\end{array}$ \\
\hline Beyls, 2003 & melody & uses cellular automata; graphical representation \\
\hline $\begin{array}{l}\text { Moroni et al., } 2000 \\
\quad \text { (Vox Populi) }\end{array}$ & melody & complex graphical representation \\
\hline Ventrella, 2008 & melody & the whole population comprises the melody \\
\hline Marques et al., 2010 & melody & minimizes evolutionary iterations \\
\hline
\end{tabular}

Table 17: References for Section 3.5.2, in order of appearance.

directly impossible to define a formal fitness function. Because of these inconveniences, many researchers have resorted to implement the fitness function with human evaluators. A common term for describing this class of EAs is musical IGA (interactive genetic algorithm ${ }^{29}$, MIGA for short). As MIGAs represent a substantial percentage of the total body of work on EAs for algorithmic composition, this subsection is devoted to them.

The first MIGAs were implemented by composers intrigued by the concept of evolutionary computing, resulting in more or less peculiar architectures from the perspective of common practice in evolutionary computing, but also by computer scientists exploring

29. Most research tagged as IGA does not use the binary genotypes commonly associated with the term genetic algorithm, but the term is very common. 
the field. Hartmann (1990), inspired by Dawkins' biomorphs, presented one of the first applications of evolutionary computing to composition, a MIGA that he unfortunately described in a notoriously laconic and obscure language, resulting in a very low citation rate for his work. Also inspired by the biomorphs, Nelson (1993) described a toy MIGA for evolving short rhythms over a fixed melodic structure, with simple binary genotypes (each bit simply denoted the presence or absence of sound). More formal models similar in scope to Nelson's were designed by Horowitz (1994) and Pazos et al. (1999). They implemented rhythm generators for multiple instruments, each one with its own independent rhythm pattern encoded in the genotype (Horowitz's genotypes were parametric, while Pazos et al. used more direct binary encodings). Degazio (1996) implemented a system in which the genotype was a set of parameters (in later iterations, a mini-language to describe the parameters) to instruct his CAAC software to generate melodies.

The best known MIGA may be GenJam, a system for generating jazz solos, developed over several years. In its first incarnation (Biles, 1994), it was formulated as a MIGA with two hierarchically structured populations: one of measures, and other of jazz phrases, constructed as sequences of measures. Given a chord progression and several other parameters, jazz solos emerged by concatenating selected phrases during the evolutionary process, and the fitness was integrated over time by accumulating fixed increments and decrements from simple good/bad indications from the evaluator. Further iterations of the system included the already discussed use of ANNs as fitness functions (Biles et al., 1996) and the possibility to trade fours with a human performer, by dynamically introducing into the population the music performed by the human (Biles, 1998). Tokui and Iba (2000) used a similar solution for creating rhythms with multiple instruments: a population of short sequences specified as list of notes, and another population of tree structures representing functions in a simple macro language that used the short sequences as building blocks. Another example of hierarchical structuring of the MIGA is Jacob's (1995) system for general-purpose composition, with three inter-dependent evolutionary processes: one involving the human user to train ears to evaluate short musical sequences, another one to compose musical phrases using the ears (filters) as fitness functions, and another also involving the human user to train an arranger that reorders the resulting phrases into the final output of the system. Schmidl (2008) implemented a similar system, but without a high-level arranger module, and with ears automatically trained from a set of examples, in order to minimize user interaction and enable real-time composition.

Genetic programming with interactive evaluation has been used several times. Putnam (1994) implemented an early example: each individual coded for a set of functions that generated a melody as the result of an iterated function system. Tokui and Iba's (2000) example has been already cited. Ando and Iba (2007) implemented a fully interactive system (not only the selection, but also the reproduction and mutation were user-guided), where the genotype model was similar to Dahlstedt's (2007). MacCallum et al. (2012) used trees to encode Perl expressions that generated polyphonic short loops, but concentrated on analyzing the interactive evolution from the point of view of theoretical biology. KaliakatsosPapakostas et al. (2012) used a rather different approach to generate 8-bit melodies: each individual was a function composed of bitwise operators that generated a waveform by iterating the function. In fact, that work might be described as sound synthesis over large time scales, rather than music composition. 
Some MIGAs with graph-based genetic representations also exist, as the implementation based in CPPNs (Hoover et al., 2012) that was already cited in Section 3.4.2. McDermott and O'Reilly (2011) used a similar paradigm: genotypes were sequences of integers that indirectly encoded graphs, whose nodes represented functions, and their connections were compositions of functions. The output nodes generated musical output in one or more voices, which were modulated by user inputs.

A problem common to all MIGAs is user fatigue: candidate solution evaluation is a comparatively slow and monotone task that rapidly leads to user fatigue. Even with small population sizes and small numbers of generations, it remains a significant problem that has been solved by many researchers in different ways. For example, Ralley (1995) used a binary representation with relative pitch encoding for the genotypes, and classified the population with a clustering algorithm, deriving similarity metrics from rudimentary spectral analysis of the scores. The user was simply required to evaluate the closest composition to the centroid of each cluster. A more exotic solution was employed by previously cited Tokui and Iba (2000): training a neural network to filter out candidates with low fitness, thus presenting to the user individuals of acceptable quality. Unehara and Onisawa (2001) presented just $10 \%$ of candidate melodies to the human user, and then parts of the genomes of the best rated ones were dispersed in the population by "horizontal gene transfer". McDermott and O'Reilly (2011) also limited the number of candidates exposed to user rating, filtering out the worst ones with heuristic functions. Another option to minimize user fatigue is to produce small compositions that already are reasonably good. The Melomics system (see last paragraph in Section 3.5.1) can be used in this way (Díaz-Jerez, 2011).

Other common ways to manage the problem include low population sizes and/or hierarchical structuring of the algorithm (Biles, 1994), or providing statistical information and/or rendering graphical representations of the compositions in order to make possible their evaluation without actually listening to them (Degazio, 1996). Graphical representations are also particularly useful if using generative methods as L-systems or cellular automata (Beyls, 2003). Putnam (1994) used a web interface to reach out to more volunteers. Moroni et al. (2000) tried to solve the problem using sophisticated GUI abstractions, with complex non-linear mappings between the graphic controls and the parameters of the fitness function and other aspects of the evolutionary process, to produce a highly modulable system for real-time interactive composition of melodies. To mitigate user fatigue, Ventrella (2008) presented a population of short melodies as a continuous stream of sound; fitness was obtained through a binary signal set by the user. Marques et al. (2010) limited user fatigue in the generation of short, simple melodies by severely limiting the number of generations of the algorithm, and generating a reasonably good starting population by drawing the notes using Zipf's law.

\subsubsection{Other Population-Based Methods}

Finally, this subsection presents other methods that are also population-based. For example, the metaheuristic method Harmony Search is inspired in the improvisation process of musicians, though in practice can be framed as an evolutionary method with a specific way to structure candidate solutions and perform selection, crossover and mutation operations. Geem and Choi (2007) used this method to harmonize Gregorian chants (i.e., to write or- 


\begin{tabular}{|c|c|c|}
\hline Reference & Composition task & Comments \\
\hline Geem \& Choi, 2007 & $\begin{array}{l}\text { harmonize } \\
\text { Gregorian chants }\end{array}$ & harmony search \\
\hline Geis \& Middendorf, 2008 & $\begin{array}{c}\text { four-part } \\
\text { harmonization }\end{array}$ & multi-objective Ant Colony Optimization \\
\hline Tominaga \& Setomoto, 2008 & $\begin{array}{l}\text { polyphony, } \\
\text { counterpoint }\end{array}$ & artificial chemistry \\
\hline Werner \& Todd, 1997 & melody & $\begin{array}{l}\text { co-evolutionary algorithm, Markov chains used } \\
\text { as evolvable fitness functions }\end{array}$ \\
\hline Bown \& Wiggins, 2005 & melody & $\begin{array}{l}\text { individuals are Markov chains } \\
\text { that compose and evaluate music }\end{array}$ \\
\hline Miranda, 2002 & melody & $\begin{array}{l}\text { individuals agree on a common set of } \\
\text { "intonation patterns" }\end{array}$ \\
\hline $\begin{array}{l}\text { Miranda et al., 2003, } \\
\text { sect. IV }\end{array}$ & melody & individuals are grammars that compose music \\
\hline McCormack, 2003b & interactive soundscape & individuals indirectly compete for users' attention \\
\hline Dahlstedt \& Nordahl, 2001 & soundscape & music emerges from collective interactions \\
\hline Beyls, 2007 & soundscape & music emerges from collective interactions \\
\hline Blackwell \& Bentley, 2002 & soundscape & music emerges from collective interactions \\
\hline Eldridge \& Dorin, 2009 & $\begin{array}{l}\text { soundscape, } \\
\text { sound synthesis }\end{array}$ & $\begin{array}{l}\text { music emerges from collective interactions, } \\
\text { individuals exist in the frequency domain }\end{array}$ \\
\hline Bown \& McCormack, 2010 & $\begin{array}{l}\text { interactive soundscape, } \\
\text { sound synthesis }\end{array}$ & $\begin{array}{l}\text { music emerges from collective interactions, } \\
\text { individuals exist in the frequency domain }\end{array}$ \\
\hline
\end{tabular}

Table 18: References for Section 3.5.3, in order of appearance.

ganum lines for the chants). Other methods are also population-based but not properly evolutionary. An example is the use of Ant Colony Optimization (ACO) to solve constraint harmonization problems (Geis \& Middendorf, 2008), already mentioned in Section 3.2.3. In $\mathrm{ACO}$, the candidate solutions are represented as paths in a graph, and a population of agents (ants) traverse the graph, cooperating to find the optimal path.

As a more exotic example, an Artificial Chemistry is a generative system consisting of a multiset of strings of symbols. These strings (analogues of molecules) can react according to a pre-specified set of rules (analogues of chemical reactions), generating new strings from the existing ones. Tominaga and Setomoto (2008) used this method, encoding polyphonic compositions in the strings and musical rules for counterpoint in the reaction rules of the artificial chemistry: starting from a set of simple strings, the system generated progressively more complex ones, though the aesthetical value of the resulting compositions varied widely.

A more popular method based on populations of individuals is the Artificial Ecosystem. In an artificial ecosystem, compositions emerge from the interaction between individuals in a simulation with evolutionary and/or cultural interactions, taking inspiration in the evolutionary origins of music in humans (Wallin \& Merker, 2001). Frequently, the complexity of the simulations is severely limited by the available computational power, and in some cases the goal is not music composition per se, but the study of evolutionary dynamics, the emergence of shared cultural traits and avant-garde artistic experimentation. 
An early example by Werner and Todd (1997) investigated sexual evolutionary dynamics in a population of males (small compositions) and females (Markov chains initially generated from a corpus of songs) that evaluated how much the males deviated from their expectations. However, most studies use just one kind of agent, as in the work of Bown and Wiggins (2005), whose agents used Markov chains for both compose and analyze music. Miranda (2002) and Miranda, Kirby, and Todd (2003, sect. IV) implemented models with a similar goal: to study the emergence of common structures (shared cultural knowledge). In the first case a population of agents strove to imitate each other's intonation patterns (short sequences of pitches); in the second case agents learned to compose music by inferring musical grammars from other agents' songs. Bosma (2005) extended Miranda's (2002) model, using neural networks in the agents to learn and compose music, although with tiny population sizes. As part of an art installation, McCormack (2003b) proposed a virtual ecosystem with evolving agents able to compose music using a rule-based system, competing for resources that were indirectly determined by the interest of human observers.

An alternative is that the music is not composed by the agents, but emerges as an epiphenomenon of the whole ecosystem. The models of Dahlstedt and Nordahl (2001) and Beyls (2007) used simple organisms in a two-dimensional space whose collective behavior was mapped into complex compositions, while Blackwell and Bentley's (2002) model was similar but three-dimensional, and the dynamics of the agents were inspired in swarm and flocking simulations. While all these examples use either homogeneous or spatially structured ecosystems, a recent trend is the use of sound as an environment in itself. In Eldridge and Dorin's (2009) model, the agents dwelt in the one-dimensional space of the Fourier transform of a sample of ambient sound, feeding off and moving the energy across frequencies. Bown and McCormack (2010) implemented a similar model, in which the agents were neural networks that generated sound and competed for room in the space of frequencies of ambient sound.

\subsection{Self-Similarity and Cellular Automata}

In the late 1970s, two notable results about music were reported by Voss and Clarke (1978). The first was that, for music of many different styles, the spectral density of the audio signal was (approximately) inversely proportional to its frequency; in other words, it approximately follows a $1 / f$ distribution. This is not so surprising: many different data series follow this property, from meteorological data to stock market prices; it is usually referred to as $1 / f$ noise or pink noise. The second result was that random compositions seemed more musical and pleasing (for a wide range of evaluators, from unskilled people to professional musicians and composers) when the pitches were determined by a source of $1 / f$ noise, rather than other common random processes as white (uncorrelated) noise or Brownian motion (random walks). Although the first result has been since challenged ${ }^{30}$, the second one has been used by composers as a source of raw material. Bolognesi (1983) implemented an early example influenced by Voss and Clarke's results, but composers used data series with $1 / f$ noise as raw material even before these results, early in the 1970s (Doornbusch, 2002).

30. The main criticism is that the data samples used by Voss and Clarke were hours long, merging in each sample many different compositions (and even non-musical sounds from a radio station). In view of their 


\begin{tabular}{|c|c|c|}
\hline Reference & Composition task & Comments \\
\hline Voss \& Clarke, 1978 & melody & first reference to $1 / f$ noise in music \\
\hline Bolognesi, 1983 & melody & early deliberate use of $1 / f$ noise in music \\
\hline Doornbusch, 2002 & melody & reference to early non-deliberate use of $1 / f$ noise in music \\
\hline Gogins, 1991 & melody & iterated function systems \\
\hline Pressing, 1988 & $\begin{array}{c}\text { melody, } \\
\text { sound synthesis }\end{array}$ & chaotic non-linear maps \\
\hline Herman, 1993 & melody & chaotic non-linear dynamical systems \\
\hline Langston, 1989 & melody & fractional Brownian motion \\
\hline Díaz-Jerez, 2000 & melody & fractals and other self-similar systems \\
\hline Bidlack, 1992 & melody & various fractal and chaotic systems \\
\hline $\begin{array}{c}\text { Leach \& Fitch, } 1995 \\
\text { (XComposer) }\end{array}$ & melody, rhythm & uses various fractal and chaotic systems \\
\hline Hinojosa-Chapel, 2003 & melody & uses various fractal and chaotic systems to fill the \\
\hline Coca et al., 2011 & melody & uses chaotic systems to add variation \\
\hline
\end{tabular}

Table 19: References for Section 3.6, in order of appearance.

There remains the question of why $1 / f$ noise produces more musical results that other random processes. The consensus in the research and artistic communities is self-similarity: the structure of $1 / f$ noise is statistically similar across several orders of magnitude (Farrell et al., 2006). Self-similarity is a common feature in classical music compositions (Hsü \& Hsü, 1991), and is also one of the defining features of fractals (in fact, $1 / f$ noise also has fractal characteristics). Because of this, fractals have been extensively used as a source of inspiration and raw material for compositions and CAAC software. In general, selfsimilar musical patterns have multiple levels of structure, with pleasing regularities but also dotted with sudden changes. Because these characteristics can be also present in the output of chaotic systems (whose attractors are also fractal structures), these are also used to generate musical patterns. Commonly used techniques to generate self-similar musical patterns include chaotic systems such as iterated function systems (Gogins, 1991), nonlinear maps (Pressing, 1988) and non-linear dynamical systems (Herman, 1993), but also fractional Brownian motion (Langston, 1989), cellular automata (discussed below) and Lsystems (already discussed in Section 3.1.1). More exotic methods are also possible, as musical renderings of fractal images or number sequences with fractal characteristics (DíazJerez, 2000). These methods are widely regarded as not suitable to produce melodies or compositions in their own right, but as a source of inspiration or raw material (Bidlack, 1992). Because of this, no extensive review will be provided here. ${ }^{31}$ However, full-fledged algorithmic composition methods can use them as part of the creative process, as in Leach and Fitch's (1995) XComposer, where chaotic systems are used to fill the structures laid

critics, such as Nettheim (1992), these samples could not possibly be representative of single musical pieces (see also the discussion in Díaz-Jerez, 2000, pp. 136-138).

31. Good (if somewhat outdated) reviews can be found in the work of Jones (1989), Díaz-Jerez (2000) and Nierhaus (2009). The list of composers using fractals and chaotic systems for CAAC is so long that it is impractical to consistently describe all the existing relevant work. 
down by a hierarchical model, or in Hinojosa-Chapel's (2003) paradigm for interactive systems, where they are also used as a source of musical material. They can also be used to add complexity to compositions generated by other means. ${ }^{32}$

\subsubsection{Cellular Automata}

A cellular automaton (CA) is a discrete (in time, space and state) dynamic system composed of very simple computational units (cells) usually arranged in an ordered n-dimensional (and potentially unbounded) grid (or any other regular tiling). Each cell can be in one of a finite number of states. In each discrete time step, each cell's state is deterministically updated, using a set of transition rules that take into account its own state and its neighbors' states. Although this definition can be generalized in multiple ways, it represents a good first approximation. Cellular automata are used in many disciplines across Science and the Humanities as dynamical models of complex spatial and temporal patterns emerging from the local interaction of many simple units; music composition is just one of these disciplines. Cellular automata can be used to generate fractal patterns and discrete versions of chaotic dynamical systems, but they also represent an alternative computational paradigm to realize algorithmic composition. ${ }^{33}$ Unfortunately, just like fractals and chaotic systems, CA also tend to produce interesting but somewhat unmusical patterns that are used as inspiration or raw material rather than directly as music compositions. Although CA are argued to be better suited to sound synthesis than to algorithmic composition (Miranda, 2007), only the latter application will be reviewed here.

Xenakis was known to be deeply interested in the application of CA to music. In his orchestral composition Horos, released in 1986, he is widely regarded to have used a CA to configure the structure of the composition, though it was then heavily edited by hand (Hoffmann, 2002). Early, better documented explorations of CA for music composition include the implementations of Beyls (1989), Millen (1990), and Hunt et al. (1991), which mapped the patterns generated by user-defined CA to MIDI output. Beyls (1989) presented CA as a generative system for real-time composition of avant-garde music, exploring several ways to complexify the generated musical patterns (as changing the transition rules according to meta-rules), while Millen (1990) presented a minimalist CAAC system. Hunt et al. (1991) implemented another CAAC system designed to give the composer more control over the composition process. Echoing Beyls's (1989) early work, Ariza (2007) proposed to bend the transition rules in order to increase the space of parameters available to the composer for experimentation, by either randomly changing the state of some isolated cells or dynamically changing the transition rules from one generation to the next.

CAMUS (Miranda, 1993) is a more known CA system for algorithmic composition with an innovative design using two bidimensional CA: Conway's Game of Life (used to determine musical sequences) and Griffeath's Crystalline Growths (used to determine the instrumentation of the notes generated by the first CA). Each activated cell in the Game of Life was mapped to a sequence of three notes, whose instrument was selected according to the corresponding cell in the second CA (the Crystalline Growths system). Unfortunately, according to its own creator (Miranda, 2007), CAMUS did not produce very musical results: its out-

32. See, e.g., the description of the work by Coca et al. (2011) in Section 3.4.

33. For a more detailed survey, see e.g. the work of Burraston and Edmonds (2005). 


\begin{tabular}{|c|c|c|}
\hline Reference & Composition task & Comments \\
\hline Hoffmann, 2002 & structure & reference to early use of CA in music (Xenakis's Horos) \\
\hline Beyls, 1989 & melody & early use of $\mathrm{CA}$ in music \\
\hline Millen, 1990 & melody & early use of CA in music \\
\hline Hunt et al., 1991 & melody & early use of $\mathrm{CA}$ in music \\
\hline Ariza, 2007 & melody & dynamically changing CA rules \\
\hline $\begin{array}{l}\text { Miranda, } 1993 \\
\text { (CAMUS) }\end{array}$ & $\begin{array}{c}\text { melody, } \\
\text { instrumentation }\end{array}$ & $\begin{array}{l}\text { two CA: one for the melody, } \\
\text { other for the instrumentation }\end{array}$ \\
\hline $\begin{array}{l}\text { McAlpine et al., } 1999 \\
\quad \text { (CAMUS 3D) }\end{array}$ & $\begin{array}{l}\text { melody, rhythm, } \\
\text { instrumentation }\end{array}$ & same as above, plus Markov chains to select rhythm \\
\hline Bilotta \& Pantano, 2001 & melody & explores several mappings from CA to music events \\
\hline $\begin{array}{l}\text { Dorin, } 2002 \\
\text { (Liquiprism) }\end{array}$ & rhythmic patterns & several interacting $\mathrm{CA}$ \\
\hline Ball, 2005, Miljkovic, 2007 & melody, rhythm & references to WolframTones \\
\hline Phon-Amnuaisuk, 2010 & melody & uses ANNs to learn CA rules \\
\hline Beyls, 2003 & melody & interactive evolutionary algorithm \\
\hline Bilotta \& Pantano, 2002 & melody & $\begin{array}{l}\text { extends Bilotta and Pantano's (2001) work } \\
\text { with an evolutionary algorithm }\end{array}$ \\
\hline Lo, 2012 & melody & $\begin{array}{c}\text { evolutionary algorithm, } \\
\text { Markov chains used in the fitness function }\end{array}$ \\
\hline
\end{tabular}

Table 20: References for Section 3.6.1, in order of appearance.

put was more properly considered as raw material to be edited by hand. CAMUS was later generalized, using a Markov chain to determine the note durations and three-dimensional versions of the Game of Life and Crystalline Growths (McAlpine et al., 1999).

More recently, Bilotta and Pantano (2001) explored several different mappings to generate music from CA: local codes (mapping cells to pitches, the usual mapping in most papers), global codes (mapping the entropy of the whole pattern in each generation to musical events) and mixed codes (mapping groups of cells to musical events). Dorin (2002) used six bidimensional finite CA arranged in a cube (their edges connected), running at different speeds, to generate complex poly-rhythmic patterns. ${ }^{34}$ Finally, WolframTones ${ }^{35}$ (Ball, 2005) is a commercial application of CA to music composition, using a database of four billions of transition rules for one-dimensional CA (all possible transition rules taking into account five neighbors). WolframTones searches for rules that produce chaotic or complex patterns. These patterns are mapped to musical events, and the system is able to search for patterns whose musical mapping resembles one of a set of pre-defined musical styles (Miljkovic, 2007).

Although CA are commonly used to generate musical material in a "uncontrolled" way (i.e., the composer tunes the parameters of the CA by hand), it is possible to use other methods to design the CA (states, transition rules, etc.). For example, Phon-Amnuaisuk

34. In Section 3.4.2, similar work (Dorin, 2000) with Boolean networks (a connectionist paradigm usually seen as a generalization of CA) was mentioned.

35. http://tones.wolfram.com/ 
(2010) used artificial neural networks trained to learn the transition rules of a CA: given a melody, its piano-roll notation was interpreted as the temporal pattern of a CA, and the network was trained to learn the transition rules for that temporal pattern. Then, given other initial conditions, the network produced new compositions in piano-roll notation. Evolutionary algorithms are also used to design the parameters (transition rules, states, etc.) of CA. In some cases, previous work with hand-designed CA is adapted to use an evolutionary algorithm. This is the case of Beyls (2003), who used an interactive evolutionary algorithm to evolve the parameters of a CA, and Bilotta and Pantano (2002), who adapted their previously discussed work (Bilotta \& Pantano, 2001) to use an evolutionary algorithm, although the fitness function was poorly described. Lo (2012) applied evolutionary algorithms to generate CA for algorithmic composition in a more comprehensive way, experimenting with various fitness functions based on extracting statistical models from a corpus of pre-existing compositions, including metrics based on Markov models and Zipf's law.

\section{Conclusions}

In this survey, several hundreds of papers on algorithmic composition have been briefly reviewed. Obviously, none of them has been described in detail. Rather, this survey has been intended as a short reference guide for the various methods commonly used for algorithmic composition. As Pearce et al. (2002) noted, most papers on algorithmic composition do not adequately (a) specify the precise practical or theoretical aims of research; (b) use a methodology to achieve these aims; or (c) evaluate the results in a controlled, measurable and repeatable way. Researchers of algorithmic composition have very diverse backgrounds, and, in many cases, they do not present their work in a way that enables comparison with others. Because of these considerations, we have presented the literature in a "narrative" style, classifying the existing work in several broad categories, and providing brief descriptions of the papers in an approximately chronological order for each category.

\subsection{About Creativity}

Algorithmic composition automates (to varying degrees) the various tasks associated with music composition, such as the generation of melodies or rhythms, harmonization, counterpoint and orchestration. These tasks can be applied in two ways: (a) to generate music imitating a corpus of compositions or a specific style, and (b) to automate composition tasks to varying degrees, from designing mere tools for human composers, to generating compositions without human intervention:

Generating music imitating a corpus of compositions or a specific style. Most instances of this kind of problem (including real-time improvisation systems that elaborate on input from human musicians) can be considered as solved: imitation problems have been tackled with many different methods, in many cases with reasonable success (such as Cope's EMI, 1992, or Pachet's Continuator, 2002). In fact, since the origins of computational algorithmic composition, there has been a bias in the research community towards imitation problems (Nierhaus, 2009). This may be attributed to the 
difficulty to merge the mindset of computer science (clear-cut definitions, precise algorithms, straight methodologies) with the mindset of artistic work (intuition, vagueness, cultural heritage and artistic influences). These two mindsets may be compared to the once common cultural divide in Artificial Intelligence between neats and scruffies. Unfortunately, while the neats reign supreme in Artificial Intelligence, they have yet to gain the upper hand in Artificial Creativity.

Automating composition tasks to varying degrees. In the case of automated systems for algorithmic composition intended to reproduce human creativity in some way, there is the problem of evaluating their performance: the concept of artistic creativity eludes a formal, unambiguous and effective definition. This makes it difficult to evaluate these systems in a completely rigorous way. Certainly, many frameworks have been proposed for assessing computational creativity ${ }^{36}$, but not one can be easily and uniformly applied to computers and humans alike, in a way that does not spark controversy. It may seem simple to measure computational creativity against human standards: we can simply ask people to listen to human and machine compositions, and declare an algorithmic composition system as creative if these people cannot tell apart its compositions from human ones. As Ariza (2009) noted, this kind of "musical Turing Test" has been performed by many different researchers trying to validate their systems, but they are valid if the algorithmic composition system just aspires to imitate, not to be truly creative and create a truly innovative work of art.

There is also the view that systems for algorithmic composition cannot attain true creativity, even in principle. In fact, it has been suggested (Kugel, 1990) that no Turing-equivalent formalism can truly simulate human creativity, i.e., musical creativity is not effectively computable, thus preventing computer systems from completely imitating human composers, even in theory. This argument is not without merits, but it is open to debate, precisely because it lacks a rigorous, unambiguous definition of creativity.

\subsection{About the Methods}

Regardless of these (more or less abstract) considerations about true creativity, this survey has presented existing work on algorithmic composition, organized in several categories. As described at the beginning of Section 3, these categories can be grouped in a few classes:

Symbolic AI (grammars and rule-based systems). Under this umbrella, we have grouped very different techniques. These techniques can be used both for imitation (be it the style of a specific composer, or more generally a musical style) and automation of composition tasks. They have proved very effective, and they are very popular (at least, by sheer volume of reviewed work), but in most cases, they are very labor-intensive, because they require musical knowledge to be encoded and maintained in the symbolic framework of choice. There has also been a clear trend towards more and more formal systems, gradually moving from ad hoc rule systems to constraint satisfaction and other various formalisms.

36. For example, Gero's (2000), Pearce and Wiggins's (2001), Ritchie's (2007) and Boden's (2009). 
Machine learning (Markov chains and artificial neural networks). Because of their nature, machine learning techniques are used primarily for imitation, although both Markov chains (and related statistical methods) and artificial neural networks can be also used to automate composition tasks (such as harmonization). It should be noted that some techniques described here as symbolic AI are also machine learning (like Cope's ATNs, rule learning or case-based reasoning).

Optimization techniques (evolutionary algorithms). As in the case of machine learning, optimization techniques (mostly evolutionary algorithms) have been profusely used for imitation, since it is natural to express the objective of the optimization (the fitness function) as the distance to the musical style to be imitated. However, the automation of composition tasks has also been explored, more so than in the case of machine learning techniques.

Self-similarity and cellular automata. Strictly speaking, these techniques are not a form of AI. As explained at the beginning of Section 3, they just represent a convenient way to generate novel musical material without resorting to human musical knowledge, but the problem is that musical material generated in this way is very rough; it is most commonly used by human composers as raw material to build upon.

After reviewing the literature, it becomes apparent that there is no silver bullet: except for strict, limited imitation of specific musical styles or real-time improvisation systems that elaborate on input from human musicians, almost all approaches to algorithmic composition seem to be unable to produce content which can be deemed on a par with professional human composers, even without taking into account the problem of creativity as discussed in Section 4.1. Very few examples stand out, and then only in some niche applications, such as the contemporary classical music composed by Iamus (Ball, 2012).

As there is no silver bullet, one obvious way forward is the hybridization of two or more methods. In fact, from our review of the existing work, it seems apparent that many researchers are already following this route, but there are some hybridizations that have rarely been explored. For example, the music material produced by systems based on self-similarity and CA is commonly regarded as a mere source of inspiration for human composers, rather than as a proper way to automate the composition of music, because this music material generally lacks structure. However, it can be used as the first stage in a process of algorithmic composition, to be modified and refined by subsequent stages, probably based on some form of machine learning if the goal is to produce music in a specific musical style, or some knowledge-based system (such as Leach and Fitch's XComposer, 1995). In the case of evolutionary algorithms, self-similarity systems may be used to seed the initial population, or to introduce variety to avoid premature convergence, and CA may be used as individuals to be evolved (such as in the work of Lo, 2012, which also features machine learning techniques). More research is required to explore the potential of this kind of approach, combining self-similarity and CA-based systems with other methods.

In the case of optimization techniques, the multi-objective paradigm has rarely been used, at least in comparison with the traditional single-objective approach. Composing music usually requires balancing a set of many different, sometimes conflicting objectives, to configure the various aspects of the music, so multi-objective optimization seems a natural 
way to tackle this problem. All too often, researchers use a weighted sum of parameters to conflate all these objectives into a single fitness function. Very few researchers use multi-objective optimization, as do Geis and Middendorf (2008), Carpentier and Bresson (2010), De Prisco et al. (2010), and Freitas and Guimarães (2011). While multi-objective optimization is harder (both conceptually and in practice), it represents a natural way of dealing with the complexity of having many different objectives, and it should be explored more by the research community.

Finally, in the specific case of evolutionary algorithms, the issue of encoding the individuals should also be examined. Looking at algorithmic composition as an optimization problem, search spaces for musical compositions tend to be huge and high-dimensional. Direct encodings (such as directly representing music as a sequence of pitches) make it very difficult to explore the search space in an effective way, with problems of scalability (the performance degrades significantly as the size of the problem increases) and solution structure (the solutions generated by the algorithm tend to be unstructured, hard to adapt and fragile). This problem is mitigated by indirect encodings, in which the genotype does not directly represent the phenotype, but rather a "list of instructions" to build it. Many different types of indirect encoding have been used in algorithmic composition, such as L-systems, other types of grammars, or the various encoding styles used in genetic programming. However, other advanced techniques for indirect encoding have rarely been applied to algorithmic composition, in order to overcome the aforementioned problems of scalability and solution structure, such as those related to artificial embryogenies (Stanley \& Miikkulainen, 2003), which have been inspired by biological developmental processes. Adding these to the evolutionary toolkit may be a way to enable more and more complex compositional tasks to be tackled.

\subsection{Final Thoughts}

Computers have come to stay: the use of CAAC software is prevalent among many composers, and some artistic scenes (as generative music) embrace computer-generated music as part of their identity. However, creativity is still in the hands of composers for the most part. As argued in Section 4.1, creativity is an inherently subjective concept, and it is arguably debatable the point at which a computational system may become truly creative. However, even if a precise definition cannot be agreed upon, it is easy to see that the development of algorithmic composition systems capable of independent creativity will radically change the process of music composition, and consequently the market for music. This should not be seen as yet another case of computers replacing humans in an ever more sophisticated activity, but a potentially radical disruption in the way composers perform their work: just like a pedagogical expert system does not supersedes the role of human teachers, but enable new ways to do their work.

Being music one of the arts with a stronger mathematical background, it is not surprising that most of the debate on whether machines can make original and creative works has centered in this subfield of computational creativity. Hybridization of different techniques, bioinspiration, and the use of high performance computing might bring about new realms of (computer-) creativity. As science writer Philip Ball put it in his analysis of Melomics' 
music composition technology: "... unfolding complex structure from a mutable core has enabled the kind of dramatic invention found in biological evolution" (Ball, 2012).

\section{Acknowledgments}

The authors wish to thank Ilias Bergstrom for his comments on a preliminary version of the manuscript. Also, the critical review of our anonymous referees has greatly improved the final version. This study was partially supported by a grant for the MELOMICS project (IPT-300000-2010-010) from the Spanish Ministerio de Ciencia e Innovación, and a grant for the CAUCE project (TSI-090302-2011-8) from the Spanish Ministerio de Industria, Turismo y Comercio. The first author was supported by a grant for the GENEX project (P09-TIC5123) from the Consejería de Innovación y Ciencia de Andalucía. The first author also wishes to thank his wife Elisa and his daughter Isabel for being there day after day, in spite of the long hours spent writing this manuscript, and his family for the invaluable support they have provided.

\section{References}

Adiloglu, K., \& Alpaslan, F. N. (2007). A machine learning approach to two-voice counterpoint composition. Knowledge-Based Systems, 20(3), 300-309.

Aguilera, G., Galán, J. L., Madrid, R., Martínez, A. M., Padilla, Y., \& Rodríguez, P. (2010). Automated generation of contrapuntal musical compositions using probabilistic logic in Derive. Mathematics and Computers in Simulation, 80(6), 1200-1211.

Alfonseca, M., Cebrián, M., \& Ortega, A. (2005). Evolving computer-generated music by means of the normalized compression distance. In Proceedings of the WSEAS International Conference on Simulation, Modelling and Optimization, pp. 343-348, Stevens Point, Wisconsin, USA.

Allan, M. (2002). Harmonising chorales in the style of Johann Sebastian Bach. Master's thesis, University of Edinburgh.

Allombert, A., Assayag, G., Desainte-Catherine, M., \& Rueda, C. (2006). Concurrent constraints models for interactive scores. In Proceedings of the Sound and Music Computing Conference.

Ames, C. (1987). Automated composition in retrospect: 1956-1986. Leonardo, 20(2), 169185.

Ames, C. (1989). The Markov process as a compositional model: A survey and tutorial. Leonardo, 22(2), 175-187.

Ames, C., \& Domino, M. (1992). Understanding music with AI, chap. Cybernetic composer: an overview, pp. 186-205. The MIT Press, Cambridge.

Amiot, E., Noll, T., Agon, C., \& Andreatta, M. (2006). Fourier oracles for computer-aided improvisation. In Proceedings of the International Computer Music Conference.

Anders, T. (2007). Composing Music by Composing Rules: Design and Usage of a Generic Music Constraint System. Ph.D. thesis, Queen's University Belfast. 
Anders, T., \& Miranda, E. R. (2009). A computational model that generalises Schoenberg's guidelines for favourable chord progressions. In Proceedings of the Sound and Music Computing Conference.

Anders, T., \& Miranda, E. R. (2011). Constraint programming systems for modeling music theories and composition. ACM Computing Surveys, 43(4), 30:1-30:38.

Ando, D., \& Iba, H. (2007). Interactive composition aid system by means of tree representation of musical phrase. In Proceedings of the IEEE Conference on Evolutionary Computation, pp. 4258-4265.

Ariza, C. (2005a). Navigating the landscape of computer aided algorithmic composition systems: A definition, seven descriptors, and a lexicon of systems and research. In Proceedings of the International Computer Music Conference.

Ariza, C. (2005b). An Open Design for Computer-Aided Algorithmic Music Composition: athenaCL. Ph.D. thesis, New York University.

Ariza, C. (2006). Beyond the transition matrix: A language-independent, string-based input notation for incomplete, multiple-order, static Markov transition values. Unpublished manuscript.

Ariza, C. (2007). Automata bending: Applications of dynamic mutation and dynamic rules in modular One-Dimensional cellular automata. Computer Music Journal, 31(1), $29-49$.

Ariza, C. (2009). The interrogator as critic: The Turing test and the evaluation of generative music systems. Computer Music Journal, 33(2), 48-70.

Ariza, C. (2011). Two pioneering projects from the early history of computer-aided algorithmic composition. Computer Music Journal, 35(3), 40-56.

Aschauer, D. (2008). Algorithmic composition. Master's thesis, Vienna University of Technology.

Assayag, G., \& Dubnov, S. (2004). Using factor oracles for machine improvisation. Soft Computing - A Fusion of Foundations, Methodologies and Applications, 8(9), 604-610.

Assayag, G., Rueda, C., Laurson, M., Agon, C., \& Delerue, O. (1999). Computer-Assisted composition at IRCAM: From PatchWork to OpenMusic. Computer Music Journal, 23(3), 59-72.

Baeten, J. C. M. (2005). A brief history of process algebra. Theoretical Computer Science, $335(2-3), 131-146$.

Baggi, D. L. (1991). Neurswing: an intelligent workbench for the investigation of swing in jazz. Computer, $24(7), 60-64$.

Balaban, M., Ebcioğlu, K., \& Laske, O. E. (1992). Understanding music with AI : perspectives on music cognition. The MIT Press, Cambridge.

Ball, P. (2005). Making music by numbers online. Nature News Online (http://dx.doi.org/10.1038/050919-14).

Ball, P. (2012). Computer science: Algorithmic rapture. Nature, $488(7412), 458$. 
Baroni, M., \& Jacoboni, C. (1978). Proposal for a grammar of melody : The Bach chorales. Les Presses de l'Université de Montréal.

Bel, B. (1992). Modelling improvisatory and compositional processes. Languages of Design, Formalisms for Word, Image and Sound, 1, 11-26.

Bellgard, M. I., \& Tsang, C. P. (1992). Harmonizing music using a network of Boltzmann machines. In Proceedings of the Annual Conference of Artificial Neural Networks and their Applications, pp. 321-332, France.

Berg, P. (2011). Using the AC Toolbox. Institute of Sonology, Royal Conservatory, The Hague.

Beyls, P. (1989). The musical universe of cellular automata. In Proceedings of the International Computer Music Conference, pp. 34-41.

Beyls, P. (2003). Selectionist musical automata: Integrating explicit instruction and evolutionary algorithms. In Proceedings of the Brazilian Symposium on Computer Music.

Beyls, P. (2007). Interaction and self-organisation in a society of musical agents. In Proceedings of the European Conference on Artificial Life.

Bidlack, R. (1992). Chaotic systems as simple (but complex) compositional algorithms. Computer Music Journal, 16(3), 33-47.

Biles, J. A. (1994). GenJam: A genetic algorithm for generating jazz solos. In Proceedings of the International Computer Music Conference.

Biles, J. A. (1998). Interactive GenJam: Integrating real-time performance with a genetic algorithm. In Proceedings of the International Computer Music Conference.

Biles, J. A., Anderson, P., \& Loggi, L. (1996). Neural network fitness functions for a musical IGA. In Proceedings of the International Symposium on Intelligent Industrial Automation and Soft Computing.

Bilotta, E., \& Pantano, P. (2001). Artificial life music tells of complexity. In Proceedings of the European Conference on Artificial Life.

Bilotta, E., \& Pantano, P. (2002). Synthetic harmonies: An approach to musical semiosis by means of cellular automata. Leonardo, 35(2), 153-159.

Birchfield, D. A. (2003). Evolving intelligent musical materials. Ph.D. thesis, Columbia University, New York.

Biyikoglu, K. M. (2003). A Markov model for chorale harmonization. In Proceedings of the Triennial ESCOM Conference.

Blackwell, T. M., \& Bentley, P. (2002). Improvised music with swarms. In Proceedings of the IEEE Conference on Evolutionary Computation, pp. 1462-1467.

Boden, M. A. (2009). Computer models of creativity. AI Magazine, 30 (3), 23-34.

Boenn, G., Brain, M., De Vos, M., \& Fitch, J. (2008). Automatic composition of melodic and harmonic music by Answer Set Programming. In Proceedings of the International Conference on Logic Programming, pp. 160-174.

Bolognesi, T. (1983). Automatic composition: Experiments with self-similar music. Computer Music Journal, 7(1), 25-36. 
Bosma, M. (2005). Musicology in a virtual world: A bottom up approach to the study of musical evolution. Master's thesis, University of Groningen and University of Plymouth.

Boulanger, R. C. (Ed.). (2000). The Csound Book: Perspectives in Software Synthesis, Sound Design, Signal Processing, and Programming. The MIT Press.

Bown, O., \& McCormack, J. (2010). Taming nature: tapping the creative potential of ecosystem models in the arts. Digital Creativity, 21 (4), 215-231.

Bown, O., \& Wiggins, G. A. (2005). Modelling musical behaviour in a cultural-evolutionary system. In Proceedings of the International Joint Conference on Artificial Inteligence.

Brooks, F. P., Hopkins, A. L., Neumann, P. G., \& Wright, W. V. (1957). An experiment in musical composition. IRE Transactions on Electronic Computers, EC-6(3), 175-182.

Browne, T. M., \& Fox, C. (2009). Global Expectation-Violation as fitness function in evolutionary composition. In Proceedings of the Conference on Applications of Evolutionary Computation, pp. 538-546.

Bryden, K. (2006). Using a Human-in-the-Loop evolutionary algorithm to create DataDriven music. In Proceedings of the IEEE Conference on Evolutionary Computation, pp. 2065-2071.

Bulley, J., \& Jones, D. (2011). Variable 4: A dynamical composition for weather systems. In Proceedings of the International Computer Music Conference.

Burns, K. (1994). The History and Development of Algorithms in Music Composition, 1957-1993. Ph.D. thesis, Ball State University.

Burraston, D., \& Edmonds, E. (2005). Cellular automata in generative electronic music and sonic art: a historical and technical review. Digital Creativity, 16(3), 165-185.

Burton, A. R. (1998). A Hybrid Neuro-Genetic Pattern Evolution System Applied to Musical Composition. Ph.D. thesis, University of Surrey.

Burton, A. R., \& Vladimirova, T. (1999). Generation of musical sequences with genetic techniques. Computer Music Journal, 23(4), 59-73.

Buttram, T. (2003). DirectX 9 Audio Exposed: Interactive Audio Development, chap. Beyond Games: Bringing DirectMusic into the Living Room. Wordware Publishing Inc.

Carpentier, G., \& Bresson, J. (2010). Interacting with symbol, sound, and feature spaces in orchidée, a computer-aided orchestration environment. Computer Music Journal, $34(1), 10-27$.

Chemillier, M. (2004). Toward a formal study of jazz chord sequences generated by Steedman's grammar. Soft Computing - A Fusion of Foundations, Methodologies and Applications, 8(9), 617-622.

Chemillier, M., \& Truchet, C. (2001). Two musical CSPs. In Proceedings of the International Conference on Principles and Practice of Constraint Programming.

Chen, C. C. J., \& Miikkulainen, R. (2001). Creating melodies with evolving recurrent neural networks. In Proceedings of the International Joint Conference on Neural Networks, pp. 2241-2246. 
Chusid, I. (1999). Beethoven-in-a-box: Raymond scott's electronium. Contemporary Music Review, 18(3), 9-14.

Coca, A. E., Romero, R. A. F., \& Zhao, L. (2011). Generation of composed musical structures through recurrent neural networks based on chaotic inspiration. In Proceedings of the International Joint Conference on Neural Networks, pp. 3220-3226.

Coghlan, A. (2012). Computer composer honours Turing's centenary. New Scientist, $215(2872), 7$.

Cohen, J. E. (1962). Information theory and music. Behavioral Science, 7(2), 137-163.

Collins, N. (2009). Musical form and algorithmic composition. Contemporary Music Review, 28(1), 103-114.

Conklin, D. (2003). Music generation from statistical models. In Proceedings of the Symposium on Artificial Intelligence and Creativity in Arts and Science.

Conklin, D., \& Witten, I. (1995). Multiple viewpoint systems for music prediction. Journal of New Music Research, 24(1), 51-73.

Cope, D. (1992). Computer modeling of musical intelligence in EMI. Computer Music Journal, 16(2), 69-83.

Cope, D. (2000). The Algorithmic Composer. A-R Editions.

Cope, D. (2005). Computer Models of Musical Creativity. The MIT Press, Cambridge.

Courtot, F. (1990). A constraint-based logic program for generating polyphonies. In Proceedings of the International Computer Music Conference, pp. 292-294.

Cruz-Alcázar, P. P., \& Vidal-Ruiz, E. (1998). Learning regular grammars to model musical style: Comparing different coding schemes. In Proceedings of the International Colloquium on Grammatical Inference, pp. 211-222.

Dahlstedt, P. (2007). Autonomous evolution of complete piano pieces and performances. In Proceedings of the European Conference on Artificial Life.

Dahlstedt, P., \& Nordahl, M. G. (2001). Living melodies: Coevolution of sonic communication. Leonardo, 34 (3), 243-248.

Dalhoum, A. A., Alfonseca, M., Cebrián, M., Sánchez-Alfonso, R., \& Ortega, A. (2008). Computer-generated music using grammatical evolution. In Proceedings of the MiddleEast Simulation Multiconference, pp. 55-60.

Davismoon, S., \& Eccles, J. (2010). Combining musical constraints with Markov transition probabilities to improve the generation of creative musical structures. In Proceedings of the European Conference on the Applications of Evolutionary Computation.

De Prisco, R., Zaccagnino, G., \& Zaccagnino, R. (2010). EvoBassComposer: a multiobjective genetic algorithm for 4-voice compositions. In Proceedings of the Genetic and Evolutionary Computation Conference.

Degazio, B. (1996). The evolution of musical organisms. Leonardo Music Journal, 7, 27-33.

Díaz-Jerez, G. (2000). Algorithmic Music Using Mathematical Models. Ph.D. thesis, Manhattan School of Music. 
Díaz-Jerez, G. (2011). Composing with Melomics: Delving into the computational world for musical inspiration. Leonardo Music Journal, 21, 13-14.

Dobrian, C. (1993). Music and artificial intelligence. Unpublished manuscript. Available at http://music.arts.uci.edu/dobrian/CD.music.ai.htm.

Doornbusch, P. (2002). A brief survey of mapping in algorithmic composition. In Proceedings of the International Computer Music Conference.

Dorin, A. (2000). Boolean networks for the generation of rhythmic structure. In Proceedings of the Australasian Computer Music Conference, pp. 38-45.

Dorin, A. (2002). Liquiprism : Generating polyrhythms with cellular automata. In Proceedings of the International Conference on Auditory Display.

Drewes, F., \& Högberg, J. (2007). An algebra for tree-based music generation. In Proceedings of the International Conference on Algebraic Informatics, pp. 172-188.

Dubnov, S., Assayag, G., Lartillot, O., \& Bejerano, G. (2003). Using machine-learning methods for musical style modeling. Computer, 36(10), 73-80.

DuBois, R. L. (2003). Applications of Generative String-Substitution Systems in Computer Music. Ph.D. thesis, Columbia University.

Duff, M. O. (1989). Backpropagation and Bach's 5th cello suite (Sarabande). In Proceedings of the International Joint Conference on Neural Networks, p. 575.

Ebcioğlu, K. (1980). Computer counterpoint. In Proceedings of the International Computer Music Conference.

Ebcioğlu, K. (1988). An expert system for harmonizing four-part chorales. Computer Music Journal, 12(3), 43-51.

Edwards, M. (2011). Algorithmic composition: computational thinking in music. Communications of the ACM, 54 (7), 58-67.

Eigenfeldt, A., \& Pasquier, P. (2010). Realtime generation of harmonic progressions using controlled Markov selection. In Proceedings of the International Conference on Computational Creativity.

Eldridge, A., \& Dorin, A. (2009). Filterscape: Energy recycling in a creative ecosystem. In Proceedings of the Conference on Applications of Evolutionary Computation.

Eno, B. (1996). Generative Music, speech at the Imagination Conference. Available at http://www . inmotionmagazine.com/eno1.html.

Espí, D., Ponce de León, P. J., Pérez-Sancho, C., Rizo, D., nesta, J. I., Moreno-Seco, F., \& Pertusa, A. (2007). A cooperative approach to style-oriented music composition. In Proceedings of the International Joint Conference on Artificial Inteligence.

Farbood, M., \& Schoner, B. (2001). Analysis and synthesis of Palestrina-style counterpoint using Markov chains. In Proceedings of the International Computer Music Conference.

Farnell, A. (2007). An introduction to procedural audio and itsapplication in computer games. In Proceedings of the Audio Mostly Conference.

Farrell, S., Jan Wagenmakers, E., \& Ratcliff, R. (2006). 1/f noise in human cognition: Is it ubiquitous, and what does it mean?. Psychonomic Bulletin 8 Review, 13(4), 737-741. 
Feulner, J., \& Hörnel, D. (1994). MELONET: neural networks that learn harmony-based melodic variations. In Proceedings of the International Computer Music Conference, pp. 121-124, San Francisco.

Fox, C. W. (2006). Genetic hierarchical music structures. In Proceedings of the International Florida Artificial Research Society Conference.

Franklin, J. (2001). Multi-phase learning for jazz improvisation and interaction. In Proceedings of the Biennial Symposium on Arts and Technology.

Freitas, A., \& Guimarães, F. (2011). Melody harmonization in evolutionary music using multiobjective genetic algorithms. In Proceedings of the Sound and Music Computing Conference.

Fry, C. (1984). Flavors band: A language for specifying musical style. Computer Music Journal, 8(4), 20-34.

Garay Acevedo, A. (2004). Fugue composition with counterpoint melody generation using genetic algorithms. In Proceedings of the International Conference on Computer Music Modeling and Retrieval, pp. 96-106.

Gartland-Jones, A. (2002). Can a genetic algorithm think like a composer?. In Proceedings of the Generative Art Conference.

Geem, Z. W., \& Choi, J. Y. (2007). Music composition using harmony search algorithm. In Proceedings of the Conference on Applications of Evolutionary Computation, pp. 593-600.

Geis, M., \& Middendorf, M. (2008). Creating melodies and baroque harmonies with ant colony optimization. International Journal of Intelligent Computing and Cybernetics, $1(2), 213-218$.

Gero, J. S. (2000). Computational models of innovative and creative design processes. Technological Forecasting and Social Change, 64 (2-3), 183-196.

Gibson, P. M., \& Byrne, J. A. (1991). NEUROGEN, musical composition using genetic algorithms and cooperating neural networks. In Proceedings of the International Conference on Artificial Neural Networks, pp. 309-313.

Gill, S. (1963). A technique for the composition of music in a computer. The Computer Journal, 6(2), 129-133.

Gillick, J., Tang, K., \& Keller, R. M. (2009). Learning jazz grammars. In Proceedings of the Sound and Music Computing Conference, pp. 125-130.

Gjerdingen, R. (1988). Explorations in Music, the Arts, and Ideas: Essays in Honor of Leonard B. Meyer, chap. Concrete musical knowledge and a computer program for species counterpoint, pp. 199-228. Pendragon Press.

Gogins, M. (1991). Iterated functions systems music. Computer Music Journal, 15(1), $40-48$.

Gogins, M. (2006). Score generation in voice-leading and chord spaces. In Proceedings of the International Computer Music Conference. 
Goldman, C., Gang, D., Rosenschein, J., \& Lehmann, D. (1996). NETNEG: a hybrid interactive architecture for composing polyphonic music in real time. In Proceedings of the International Computer Music Conference, pp. 133-140.

Grachten, M. (2001). JIG : jazz improvisation generator. In Proceedings of the Workshop on Current Research Directions in Computer Music, pp. 1-6. Audiovisual Institute-UPF.

Gwee, N. (2002). Complexity and Heuristics in Rule-Based Algorithmic Music Composition. Ph.D. thesis, Louisiana State University.

Hamanaka, M., Hirata, K., \& Tojo, S. (2008). Melody morphing method based on GTTM. In Proceedings of the International Computer Music Conference, pp. 155-158.

Harris, R. (2008). Algorithmic composition of jazz. Master's thesis, University of Bath.

Hartmann, P. (1990). Natural selection of musical identities. In Proceedings of the International Computer Music Conference.

Haus, G., \& Sametti, A. (1991). Scoresynth: a system for the synthesis of music scores based on Petri nets and a music algebra. IEEE Computer, 24(7), 56-60.

Herman, M. (1993). Deterministic chaos, iterative models, dynamical systems and their application in algorithmic composition. In Proceedings of the International Computer Music Conference.

Herremans, D., \& Sorensena, K. (2012). Composing first species counterpoint with a variable neighbourhood search algorithm. Journal of Mathematics and the Arts, 6(4), 169-189.

Hild, H., Feulner, J., \& Menzel, D. (1992). HARMONET: a neural net for harmonising chorales in the style of J.S. Bach. In Proceedings of the Conference on Neural Information Processing Systems.

Hiller, L. A., \& Isaacson, L. M. (1958). Musical composition with a High-Speed digital computer. Journal of the Audio Engineering Society, 6(3), 154-160.

Hinojosa-Chapel, R. (2003). Realtime algorithmic music systems from fractals and chaotic functions: Toward an active musical instrument. Master's thesis, Universitat Pompeu Fabra.

Hirata, K., \& Aoyagi, T. (1988). How to realize jazz feelings: a logic programming approach. In Proceedings of the International Conference on Fifth Generation Computer Systems.

Hoffmann, P. (2002). Towards an "automated art": Algorithmic processes in xenakis' compositions. Contemporary Music Review, 21(2-3), 121-131.

Holm, F. (1990). CESAM: A concept engine for synthesis of audio and music. In Proceedings of the International Computer Music Conference.

Holtzman, S. R. (1981). Using generative grammars for music composition. Computer Music Journal, 5(1), 51-64.

Hoover, A. K., Szerlip, P. A., Norton, M. E., Brindle, T. A., Merritt, Z., \& Stanley, K. O. (2012). Generating a complete multipart musical composition from a single monophonic melody with functional scaffolding. In Proceedings of the International Conference on Computational Creativity. 
Hörnel, D., \& Degenhardt, P. (1997). A neural organist improvising baroque-style melodic variations. In Proceedings of the International Computer Music Conference, pp. 430433.

Hörnel, D., \& Ragg, T. (1996). A connectionist model for the evolution of styles of harmonization. In Proceedings of the International Conference on Music Perception and Cognition.

Horner, A., \& Ayers, L. (1995). Harmonization of musical progressions with genetic algorithms. In Proceedings of the International Computer Music Conference.

Horner, A., \& Goldberg, D. E. (1991). Genetic algorithms and computer-assisted music composition. In Proceedings of the International Conference on Genetic Algorithms, pp. 337-441.

Horowitz, M. D. (1994). Generating rhythms with genetic algorithms. In Proceedings of the AAAI National Conference on Artificial intelligence, Menlo Park.

Horowitz, M. D. (1995). Representing musical knowledge. Ph.D. thesis, Columbia University.

Hsü, K. J., \& Hsü, A. (1991). Self-similarity of the "1/f noise" called music. Proceedings of the National Academy of Sciences of the United States of America, 88(8), 3507-3509.

Hunt, A., Kirk, R., \& Orton, R. (1991). Musical applications of a cellular automata workstation. In Proceedings of the International Computer Music Conference.

Jacob, B. L. (1995). Composing with genetic algorithms. In Proceedings of the International Computer Music Conference.

Jensen, J. H. (2011). Evolutionary music composition: A quantitative approach. Master's thesis, Norwegian University of Science and Technology.

Johanson, B., \& Poli, R. (1998). GP-music: An interactice genetic programming system for music generation with automated fitness raters. In Proceedings of the Annual Conference on Genetic Programming, pp. 181-186.

Johnson, M., Tauritz, D. R., \& Wilkerson, R. (2004). Evolutionary computation applied to melody generation. In Proceedings of the Artificial Neural Networks in Engineering (ANNIE) Conference.

Jones, K. (1980). A space grammar for the stochastic generation of Multi-Dimensional structures. In Proceedings of the International Computer Music Conference.

Jones, K. (1981). Compositional applications of stochastic processes. Computer Music Journal, 5(2), 45-61.

Jones, K. (1989). Generative models in computer-assisted musical composition. Contemporary Music Review, 3(1), 177-196.

Kaliakatsos-Papakostas, M. A., Epitropakis, M. G., Floros, A., \& Vrahatis, M. N. (2012). Interactive evolution of 8-Bit melodies with genetic programming towards finding aesthetic measures for sound evolutionary and biologically inspired music, sound, art and design. In Proceedings of the International Conference on Evolutionary and Biologically Inspired Music, Sound, Art and Design, pp. 141-152. 
Keller, R. M., \& Morrison, D. R. (2007). A grammatical approach to automatic improvisation. In Proceedings of the Sound and Music Computing Conference, pp. 330-337.

Khalifa, Y. M. A., Khan, B. K., Begovic, J., Wisdom, A., \& Wheeler, A. M. (2007). Evolutionary music composer integrating formal grammar. In Proceedings of the Genetic and Evolutionary Computation Conference, pp. 2519-2526, New York.

Kippen, J., \& Bel, B. (1989). The identification and modelling of a percussion language, and the emergence of musical concepts in a machine-learning experimental set-up. Computers and the Humanities, 23(3), 199-214.

Kirke, A., \& Miranda, E. (2009). A survey of computer systems for expressive music performance. ACM Computing Surveys, 42(1), 3:1-3:41.

Kitani, K. M., \& Koike, H. (2010). ImprovGenerator: Online grammatical induction for onthe-fly improvisation accompaniment. In Proceedings of the International Conference on New Interfaces for Musical Expression.

Klinger, R., \& Rudolph, G. (2006). Evolutionary composition of music with learned melody evaluation. In Proceedings of the WSEAS International Conference on Computational Intelligence, Man-Machine Systems and Cybernetics, pp. 234-239, Stevens Point, Wisconsin, USA.

Kohonen, T., Laine, P., Tiits, K., \& Torkkola, K. (1991). Music and Connectionism, chap. A Nonheuristic Automatic Composing Method, pp. 229-242. The MIT Press, Cambridge.

Kugel, P. (1990). Myhill's Thesis: There's more than computing in musical thinking. Computer Music Journal, 14 (3), 12-25.

Laine, P. (2000). A Method for Generating Musical Motion Patterns. Ph.D. thesis, University of Helsinki.

Laine, P., \& Kuuskankare, M. (1994). Genetic algorithms in musical style oriented generation. In Proceedings of the IEEE Conference on Evolutionary Computation, pp. $858-862$.

Langston, P. (1989). Six techniques for algorithmic music composition. In Proceedings of the International Computer Music Conference.

Laurson, M., \& Kuuskankare, M. (2000). Towards idiomatic instrumental writing: A constraint based approach. In Proceedings of the Annual Symposium on Systems Research in the Arts.

Leach, J., \& Fitch, J. (1995). Nature, music, and algorithmic composition. Computer Music Journal, 19(2), 23-33.

Lerdahl, F., Jackendoff, R., \& Jackendoff, R. S. (1983). A Generative Theory of Tonal Music. The MIT Press, Cambridge.

Levitt, D. A. (1981). A melody description system for jazz improvisation. Master's thesis, Massachusetts Institute of Technology.

Lewis, J. P. (1991). Music and Connectionism, chap. Creation by refinement and the problem of algorithmic music composition. The MIT Press, Cambridge. 
Lidov, D., \& Gabura, J. (1973). A melody writing algorithm using a formal language model. Computer Studies in the Humanities and Verbal Behavior, 4(3-4), 138-148.

Lo, M. Y. (2012). Evolving Cellular Automata for Music Composition with Trainable Fitness Functions. Ph.D. thesis, University of Essex.

Lo, M., \& Lucas, S. M. (2006). Evolving musical sequences with N-Gram based trainable fitness functions. In Proceedings of the IEEE Conference on Evolutionary Computation, pp. 601-608.

Löthe, M. (1999). Knowledge based automatic composition and variation of melodies for minuets in early classical style. In Proceedings of the Annual German Conference on Artificial Intelligence, pp. 159-170.

Loy, G., \& Abbott, C. (1985). Programming languages for computer music synthesis, performance, and composition. ACM Computing Surveys, 17(2), 235-265.

Lozano, L., Medaglia, A. L., \& Velasco, N. (2009). Generation of Pop-Rock chord sequences using genetic algorithms and variable neighborhood search. In Proceedings of the Conference on Applications of Evolutionary Computation, pp. 573-578.

Lyon, D. (1995). Using stochastic Petri nets for real-time Nth-order stochastic composition. Computer Music Journal, 19(4), 13-22.

MacCallum, R. M., Mauch, M., Burt, A., \& Leroi, A. M. (2012). Evolution of music by public choice. Proceedings of the National Academy of Sciences of the United States of America, 109(30), 12081-12086.

Maddox, T., \& Otten, J. (2000). Using an evolutionary algorithm to generate Four-Part 18th century harmony. In Proceedings of the WSEAS International Conference on Mathematics and Computers in Business and Economics.

Manaris, B., Hughes, D., \& Vassilandonakis, Y. (2011). Monterey mirror: Combining Markov models, genetic algorithms, and power laws. In Proceedings of the IEEE Conference on Evolutionary Computation.

Manaris, B., Roos, P., Machado, P., Krehbiel, D., Pellicoro, L., \& Romero, J. (2007). A corpus-based hybrid approach to music analysis and composition. In Proceedings of the AAAI National Conference on Artificial intelligence, pp. 839-845.

Manousakis, S. (2006). Musical L-Systems. Master's thesis, The Royal Conservatory, The Hague.

Marques, V. M., Oliveira, V., Vieira, S., \& Rosa, A. C. (2000). Music composition using genetic evolutionary algorithms. In Proceedings of the IEEE Conference on Evolutionary Computation, pp. 714-719.

Marques, V. M., Reis, C., \& Machado, J. A. T. (2010). Interactive evolutionary computation in music. In Proceedings of the IEEE International Conference on Systems, Man and Cybernetics, pp. 3501-3507.

Martin, A., Jin, C. T., \& Bown, O. (2012). Implementation of a real-time musical decisionmaker. In Proceedings of the Australasian Computer Music Conference. 
Martin, A., Jin, C. T., van Schaik, A., \& Martens, W. L. (2010). Partially observable Markov decision processes for interactive music systems. In Proceedings of the International Computer Music Conference.

Mason, S., \& Saffle, M. (1994). L-Systems, melodies and musical structure. Leonardo Music Journal, 4, 31-38.

Maurer, J. (1999). A brief history of algorithmic composition. Unpublished manuscript. Available at https://ccrma.stanford.edu/ blackrse/algorithm.html.

McAlpine, K., Miranda, E., \& Hoggar, S. (1999). Making music with algorithms: A CaseStudy system. Computer Music Journal, 23(2), 19-30.

McCartney, J. (2002). Rethinking the computer music language: SuperCollider. Computer Music Journal, 26(4), 61-68.

McCormack, J. (1996). Grammar-Based music composition. Complexity International, 3, $320-336$.

McCormack, J. (2003a). The Application of L-systems and Developmental Models to Computer Art, Animation and Music Synthesis. Ph.D. thesis, Monash University.

McCormack, J. (2003b). Evolving sonic ecosystems. Kybernetes, 32(1-2), 184-202.

McDermott, J., \& O'Reilly, U. M. (2011). An executable graph representation for evolutionary generative music. In Proceedings of the Genetic and Evolutionary Computation Conference, pp. 403-410, New York.

McGuire, K. (2006). ArpEgg: a rewriting grammar for complex arpeggios. In Proceedings of the Generative Art Conference.

McIntyre, R. A. (1994). Bach in a box: the evolution of four part baroque harmony using the genetic algorithm. In Proceedings of the IEEE Conference on Evolutionary Computation, pp. 852-857.

Melo, A. F. (1998). A connectionist model of tension in chord progressions. Master's thesis, University of Edinburgh.

Miljkovic, K. (2007). From Mathematica to live performance: Mapping simple programs to music. In Proceedings of the International Conference on Mathematics and Computation in Music.

Millen, D. (1990). Cellular automata music. In Proceedings of the International Computer Music Conference.

Miranda, E. R. (1993). Cellular automata music: An interdisciplinary project. Journal of New Music Research, 22(1), 3-21.

Miranda, E. R. (2002). Mimetic development of intonation. In Proceedings of the International Conference on Music and Artificial Intelligence.

Miranda, E. R. (2007). Evolutionary Computer Music, chap. Cellular Automata Music: From Sound Synthesis to Musical Forms, pp. 170-193. Springer-Verlag London.

Miranda, E. R., \& Biles, J. A. (Eds.). (2007). Evolutionary computer music. Springer-Verlag London. 
Miranda, E. R., Kirby, S., \& Todd, P. M. (2003). On computational models of the evolution of music: From the origins of musical taste to the emergence of grammars. Contemporary Music Review, 22(3), 91-111.

Moorer, J. A. (1972). Music and computer composition. Communications of the ACM, 15(2), 104-113.

Morales, E., \& Morales, R. (1995). Learning musical rules. In Proceedings of the International Joint Conference on Artificial Inteligence.

Morgan, N. (2007). Transformation and mapping of L-Systems data in the composition of a large-scale instrumental work. In Proceedings of the European Conference on Artificial Life.

Moroni, A., Manzolli, J., Zuben, F. V., \& Gudwin, R. (2000). Vox Populi: An interactive evolutionary system for algorithmic music composition. Leonardo Music Journal, 10, $49-54$.

Morris, D., Simon, I., \& Basu, S. (2008). Exposing parameters of a trained dynamic model for interactive music creation. In Proceedings of the AAAI National Conference on Artificial intelligence, pp. 784-791.

Mozer, M. (1991). Music and Connectionism, chap. Connectionist music composition based on melodic, stylistic, and psychophysical constraints, pp. 195-211. The MIT Press, Cambridge.

Nelson, G. L. (1993). Sonomorphs: An application of genetic algorithms to the growth and development of musical organisms. In Proceedings of the Biennial Symposium on Arts and Technology, pp. 155-169.

Nelson, G. L. (1996). Real time transformation of musical material with fractal algorithms. Computers \& Mathematics with Applications, 1, 109-116.

Nettheim, N. (1992). On the spectral analysis of melody. Journal of New Music Research, $21(2), 135-148$.

Nettheim, N. (1997). A bibliography of statistical applications in musicology. Musicology Australia, 20(1), 94-106.

Nierhaus, G. (2009). Algorithmic Composition: Paradigms of Automated Music Generation. Springer Berlin / Heidelberg.

Nishijimi, M., \& Watanabe, K. (1993). Interactive music composer based on neural networks. Fujitsu Scientific Technical Journal, 29(2), 189-192.

North, T. (1991). A technical explanation of theme and variations: A computer music work utilizing network compositional algorithms. Ex Tempore, 5(2).

Olarte, C., Rueda, C., \& Valencia, F. D. (2009). New Computational Paradigms for Computer Music, chap. Concurrent Constraint Calculi: a Declarative Paradigm for Modeling Music Systems. Editions Delatour France.

Olson, H. F. (1961). Aid to music composition employing a random probability system. Journal of the Acoustical Society of America, 33, 1163-1170. 
Ortega, A., Sánchez, R., \& Alfonseca, M. (2002). Automatic composition of music by means of grammatical evolution. In Proceedings of the Conference on APL.

Ovans, R., \& Davison, R. (1992). An interactive Constraint-Based expert assistant for music composition. In Proceedings of the Canadian Conference on Artificial Intelligence, pp. 76-81.

Özcan, E., \& Erçal, T. (2008). A genetic algorithm for generating improvised music. In Proceedings of the International Conference on Artificial Evolution, pp. 266-277.

Pachet, F. (2002). Interacting with a musical learning system: The Continuator. In Proceedings of the International Conference on Music and Artificial Intelligence, pp. 103-108.

Pachet, F., \& Roy, P. (1995). Mixing constraints and objects: a case study in automatic harmonization. In Proceedings of the Conference on Technology of Object-Oriented Languages and Systems.

Pachet, F., \& Roy, P. (2001). Musical harmonization with constraints: A survey. Constraints, $6(1), 7-19$.

Pachet, F., Roy, P., \& Barbieri, G. (2011). Finite-length Markov processes with constraints. In Proceedings of the International Joint Conference on Artificial Inteligence.

Padberg, H. A. (1964). Computer-composed canon and free-fugue. Ph.D. thesis, Saint Louis University, St. Louis.

Papadopoulos, G., \& Wiggins, G. (1998). A genetic algorithm for the generation of jazz melodies. In Proceedings of the Finnish Conference on Artificial Intelligence (STeP).

Papadopoulos, G., \& Wiggins, G. (1999). AI methods for algorithmic composition: A survey, a critical view and future prospects. In Proceedings of the Symposium on Musical Creativity, pp. 110-117.

Parikh, T. (2003). Iris: artificially intelligent real-time improvisation system. Master's thesis, Emory University.

Pazos, A., Santos del Riego, A., Dorado, J., \& Romero Caldalda, J. J. (1999). Genetic music compositor. In Proceedings of the IEEE Conference on Evolutionary Computation, pp. 885-890.

Pearce, M., Meredith, D., \& Wiggins, G. (2002). Motivations and methodologies for automation of the compositional process. Musica Scientice, 6(2), 119-147.

Pearce, M., \& Wiggins, G. (2001). Towards a framework for the evaluation of machine compositions. In Proceedings of the Symposium on Artificial Intelligence and Creativity in Arts and Science, pp. 22-32.

Peck, J. M. (2011). Explorations in algorithmic composition: Systems of composition and examination of several original works. Master's thesis, State University of New York, College at Oswego.

Pennycook, B. (1985). Computer-music interfaces: A survey. ACM Computing Surveys, $17(2), 267-289$.

Pereira, F., Grilo, C., Macedo, L., \& Cardoso, A. (1997). Composing music with case-based reasoning. In Proceedings of the Conference on Computational Models of Creative Cognition. 
Pestana, P. (2012). Lindenmayer systems and the harmony of fractals. Chaotic Modeling and Simulation, 1(1), 91-99.

Phon-Amnuaisuk, S. (2002). Control language for harmonisation process. In Proceedings of the International Conference on Music and Artificial Intelligence, pp. 155-167.

Phon-Amnuaisuk, S. (2010). Investigating music pattern formations from heterogeneous cellular automata. Journal of New Music Research, 39(3), 253-267.

Phon-Amnuaisuk, S., Law, E. H., \& Kuan, H. C. (2007). Evolving music generation with SOM-fitness genetic programming. In Proceedings of the Conference on Applications of Evolutionary Computation, pp. 557-566.

Phon-Amnuaisuk, S., Tuson, A., \& Wiggins, G. (1999). Evolving musical harmonisation. In Proceedings of the International Conference on Artificial Neural Nets and Genetic Algorithms.

Pinkerton, R. C. (1956). Information theory and melody. Scientific American, 194(2), $77-87$.

Polito, J., Daida, J. M., \& Bersano Begey, T. F. (1997). Musica ex machina: Composing 16th-Century counterpoint with genetic programming and symbiosis. In Proceedings of the International Conference on Evolutionary Programming, pp. 113-124.

Ponsford, D., Wiggins, G., \& Mellish, C. (1999). Statistical learning of harmonic movement. Journal of New Music Research, 28(2), 150-177.

Pope, S. T. (1986). Music notations and the representation of musical structure and knowledge. Perspectives of New Music, 24 (2), 156-189.

Pope, S. T. (1991). A tool for manipulating expressive and structural hierarchies in music (or: "T-R trees in the MODE: A tree editor based loosely on Fred's theory"). In Proceedings of the International Computer Music Conference.

Pope, S. T. (1993). Music Processing, chap. Music composition and editing by computer, pp. 25-72. Oxford University Press.

Pope, S. T. (1995). Fifteen years of computer-assisted composition. In Proceedings of the Brazilian Symposium on Computer Music.

Pressing, J. (1988). Nonlinear maps as generators of musical design. Computer Music Journal, 12(2), 35-46.

Prusinkiewicz, P. (1986). Score generation with L-systems. In Proceedings of the International Computer Music Conference, pp. 455-457.

Prusinkiewicz, P., \& Lindenmayer, A. (1990). The algorithmic beauty of plants. SpringerVerlag New York.

Puckette, M. (2002). Max at Seventeen. Computer Music Journal, 26(4), 31-43.

Putnam, J. (1994). Genetic programming of music. Tech. rep., New mexico institute of mining and technology.

Quick, D. (2010). Generating music using concepts from Schenkerian analysis and chord spaces. Tech. rep., Yale University. 
Rader, G. M. (1974). A method for composing simple traditional music by computer. Communications of the ACM, 17(11), 631-638.

Ralley, D. (1995). Genetic algorithms as a tool for melodic development. In Proceedings of the International Computer Music Conference, pp. 501-502.

Ramalho, G., \& Ganascia, J.-G. (1994). Simulating creativity in jazz performance. In Proceedings of the AAAI National Conference on Artificial intelligence, pp. 108-113, Menlo Park.

Ramírez, R., \& Peralta, J. (1998). A constraint-based melody harmonizer. In Proceedings of the Workshop on Constraints for Artistic Applications.

Reddin, J., McDermott, J., \& O'Neill, M. (2009). Elevated pitch: Automated grammatical evolution of short compositions applications of evolutionary computing. In Proceedings of the Conference on Applications of Evolutionary Computation, pp. 579-584.

Reisig, W. (1998). Elements of Distributed Algorithms: Modeling and Analysis with Petri Nets. Springer.

Rennie, J. (2010). Ray Kurzweil's slippery futurism. IEEE Spectrum, 47(12), 24-28.

Ribeiro, P., Pereira, F. C., Ferrand, M., \& Cardoso, A. (2001). Case-based melody generation with MuzaCazUza. In Proceedings of the Symposium on Artificial Intelligence and Creativity in Arts and Science, pp. 67-74.

Ricanek, K., Homaifar, A., \& Lebby, G. (1993). Genetic algorithm composes music. In Proceedings of the Southeastern Symposium on System Theory, pp. 223-227.

Riecken, D. (1998). WOLFGANG: "emotions" and architecture which enable learning to compose music. In Proceedings of the International Conference of the Society for Adaptive Behavior.

Ritchie, G. (2007). Some empirical criteria for attributing creativity to a computer program. Journal for Artificial Intelligence, Philosophy and Cognitive Science, 17(1), 67-99.

Roads, C. (1977). Composing grammars. In Proceedings of the International Computer Music Conference.

Roads, C. (1979). Grammars as representations for music. Computer Music Journal, 3(1), $48-55$.

Roads, C. (1985). Research in music and artificial intelligence. ACM Computing Surveys, $17(2), 163-190$.

Roads, C. (Ed.). (1992). The Music Machine: Selected Readings from Computer Music Journal. The MIT Press.

Roads, C. (2004). Microsound. The MIT Press.

Roads, C., \& Strawn, J. (Eds.). (1985). Foundations of computer music. The MIT Press.

Ross, B. J. (1995). A process algebra for stochastic music composition. In Proceedings of the International Computer Music Conference.

Rothgeb, J. (1968). Harmonizing the unfigured bass: A computational Study. Ph.D. thesis, Yale University. 
Rowe, R. (1992). Interactive Music Systems: Machine Listening and Composing. The MIT Press, Cambridge.

Rueda, C., Álvarez, G., Quesada, L. O., Tamura, G., Valencia, F., Díaz, J. F., \& Assayag, G. (2001). Integrating constraints and concurrent objects in musical applications: A calculus and its visual language. Constraints, 6(1), 21-52.

Rueda, C., Assayag, G., \& Dubnov, S. (2006). A concurrent constraints factor oracle model for music improvisation. In Proceedings of the Latin American Informatics Conference.

Rueda, C., Lindberg, M., Laurson, M., Bloch, G., \& Assayag, G. (1998). Integrating constraint programming in visual musical composition languages. In Proceedings of the Workshop on Constraints for Artistic Applications.

Sabater, J., Arcos, J., \& López de Mántaras, R. (1998). Using rules to support case-based reasoning for harmonizing melodies. In Proceedings of the AAAI Spring Symposium on Multimodal Reasoning, pp. 147-151.

Sánchez-Quintana, C., Moreno-Arcas, F., Albarracín-Molina, D., Fernández, J. D., \& Vico, F. (2013). Melomics: A case-study of AI in Spain. AI Magazine, 34(3), 99-103.

Sandred, O. (2004). Interpretation of everyday gestures - composing with rules. In Proceedings of the Music and Music Science Conference.

Sandred, O. (2010). PWMC, a constraint-solving system for generating music scores. Computer Music Journal, 34(2), 8-24.

Santos, A., Arcay, B., Dorado, J., Romero, J. J., \& Rodríguez, J. A. (2000). Evolutionary computation systems for musical composition. In Proceedings of the International Conference Acoustic and Music: Theory and Applications.

Sastry, A. (2011). N-gram modeling of tabla sequences using variable-length hidden Markov models for improvisation and composition. Master's thesis, Georgia Institute of Technology.

Scaletti, C. (2002). Computer music languages, Kyma, and the future. Computer Music Journal, 26(4), 69-82.

Schmidl, H. (2008). Pseudo-Genetic algorithmic composition. In Proceedings of the International Conference on Genetic and Evolutionary Methods.

Schottstaedt, W. (1989). Current directions in computer music research, chap. Automatic Counterpoint, pp. 199-214. The MIT Press, Cambridge.

Schulze, W. (2009). A Formal Language Theory Approach To Music Generation. Ph.D. thesis, Stellenbosch University.

Schwanauer, S. (1993). Machine models of music, chap. A learning machine for tonal composition, pp. 511-532. The MIT Press, Cambridge.

Schwanauer, S. M., \& Levitt, D. A. (1993). Machine Models of Music. The MIT Press, Cambridge.

Shao, J., McDermott, J., O'Neill, M., \& Brabazon, A. (2010). Jive: A generative, interactive, virtual, evolutionary music system applications of evolutionary computation. In Proceedings of the Conference on Applications of Evolutionary Computation, pp. $341-350$. 
Shibata, N. (1991). A neural network-based method for chord/note scale association with melodies. NEC Research and Development, 32(3), 453-459.

Simoni, M., \& Dannenberg, R. B. (2013). Algorithmic Composition: A Guide to Composing Music with Nyquist. University of Michigan Press.

Soddell, F., \& Soddell, J. (2000). Microbes and music. In Proceedings of the Pacific Rim International Conference on Artificial Intelligence.

Sowa, J. F. (1956). A machine to compose music. Instruction manual for GENIAC, Oliver Garfield Company, Inc.

Spangler, R. R. (1999). Rule-Based Analysis and Generation of Music. Ph.D. thesis, California Institute of Technology.

Spector, L., \& Alpern, A. (1994). Criticism, culture, and the automatic generation of artworks. In Proceedings of the AAAI National Conference on Artificial intelligence, pp. 3-8, Menlo Park.

Spector, L., \& Alpern, A. (1995). Induction and recapitulation of deep musical structure. In Proceedings of the International Joint Conference on Artificial Inteligence, pp. 41-48.

Stanley, K., \& Miikkulainen, R. (2003). A taxonomy for artificial embryogeny. Artificial Life, 9(2), 93-130.

Steedman, M. J. (1984). A generative grammar for jazz chord sequences. Music Perception: An Interdisciplinary Journal, 2(1), 52-77.

Steels, L. (1979). Reasoning modeled as a society of communicating experts. Master's thesis, Massachusetts Institute of Technology, Cambridge.

Steels, L. (1986). Learning the craft of musical composition. In Proceedings of the International Computer Music Conference.

Stieler, W. (2012). Die mozart-Maschine. Technology Review (German edition), 12/2012, $26-34$.

Supper, M. (2001). A few remarks on algorithmic composition. Computer Music Journal, $25(1), 48-53$.

Thom, B. (2000). BoB: an interactive improvisational music companion. In Proceedings of the International Conference on Autonomous Agents, pp. 309-316, New York.

Thomas, M. T. (1985). Vivace: A rule based AI system for composition. In Proceedings of the International Computer Music Conference, pp. 267-274.

Thomas, M. T., Chatterjee, S., \& Maimone, M. W. (1989). Cantabile: A rule-based system for composing melody. In Proceedings of the International Computer Music Conference.

Thornton, C. (2009). Hierarchical Markov modelling for generative music. In Proceedings of the International Computer Music Conference.

Thywissen, K. (1999). GeNotator: an environment for exploring the application of evolutionary techniques in computer-assisted composition. Organised Sound, 4 (2), 127-133.

Tipei, S. (1975). MP1: a computer program for music composition. In Proceedings of the Annual Music Computation Conference. 
Todd, P. M. (1989). A connectionist approach to algorithmic composition. Computer Music Journal, 13(4), 27-43.

Todd, P. M., \& Loy, D. G. (1991). Music and Connectionism. The MIT Press, Cambridge.

Toiviainen, P. (1995). Modeling the target-note technique of bebop-style jazz improvisation: an artificial neural network approach. Music Perception: An Interdisciplinary Journal, 12(4), 399-413.

Toiviainen, P. (2000). Readings in Music and Artificial Intelligence, chap. Symbolic AI versus Connectionism in Music Research, pp. 47-67. Harwood Academic Publishers.

Tokui, N., \& Iba, H. (2000). Music composition with interactive evolutionary computation. In Proceedings of the Generative Art Conference.

Tominaga, K., \& Setomoto, M. (2008). An artificial-chemistry approach to generating polyphonic musical phrases. In Proceedings of the Conference on Applications of Evolutionary Computation, pp. 463-472.

Towsey, M. W., Brown, A. R., Wright, S. K., \& Diederich, J. (2001). Towards melodic extension using genetic algorithms. Educational Technology 85 Society, 4(2), 54-65.

Triviño Rodríguez, J. L., \& Morales-Bueno, R. (2001). Using multiattribute prediction suffix graphs to predict and generate music. Computer Music Journal, 25(3), 62-79.

Truchet, C., Assayag, G., \& Codognet, P. (2003). OMClouds, a heuristic solver for musical constraints. In Proceedings of the International Conference on Metaheuristics.

Tsang, C. P., \& Aitken, M. (1991). Harmonizing music as a discipline of constraint logic programming. In Proceedings of the International Computer Music Conference.

Ulrich, J. W. (1977). The analysis and synthesis of jazz by computer. In Proceedings of the International Joint Conference on Artificial Inteligence, pp. 865-872.

Unehara, M., \& Onisawa, T. (2001). Composition of music using human evaluation. In Proceedings of the IEEE International Conference on Fuzzy Systems, pp. 1203-1206.

Ventrella, J. J. (2008). The Art of Artificial Evolution, chap. Evolving Structure in Liquid Music, pp. 269-288. Springer Berlin / Heidelberg.

Verbeurgt, K., Fayer, M., \& Dinolfo, M. (2004). A hybrid Neural-Markov approach for learning to compose music by example. In Proceedings of the Canadian Conference on Advances in Artificial Intelligence, pp. 480-484.

Visell, Y. (2004). Spontaneous organisation, pattern models, and music. Organised Sound, $9(02), 151-165$.

Voss, R. F., \& Clarke, J. (1978). "1/f noise" in music: Music from 1/f noise. Journal of the Acoustical Society of America, 63, 258-263.

Walker, W. F. (1994). A conversation-based framework for musical improvisation. Ph.D. thesis, University of Illinois.

Wallin, N. L., \& Merker, B. (2001). The Origins of Music. The MIT Press.

Waschka, R. (1999). Avoiding the fitness bottleneck: Using genetic algorithms to compose orchestral music. In Proceedings of the International Computer Music Conference, pp. 201-203. 
Watson, L. A. (2008). Algorithmic composition for flute and accompaniment. Master's thesis, University of Bath.

Werner, M., \& Todd, P. M. (1997). Too many love songs: Sexual selection and the evolution of communication. In Proceedings of the European Conference on Artificial Life.

Widmer, G. (1992). Qualitative perception modeling and intelligent musical learning. Computer Music Journal, 16(2), 51-68.

Wiggins, G. A. (1998). The use of constraint systems for musical composition. In Proceedings of the Workshop on Constraints for Artistic Applications.

Wiggins, G. A. (2008). Computer models of musical creativity: A review of computer models of musical creativity by David Cope. Literary and Linguistic Computing, 23(1), 109116.

Wilson, A. J. (2009). A symbolic sonification of L-systems. In Proceedings of the International Computer Music Conference, pp. 203-206.

Wolkowicz, J., Heywood, M., \& Keselj, V. (2009). Evolving indirectly represented melodies with corpus-based fitness evaluation. In Proceedings of the Conference on Applications of Evolutionary Computation, pp. 603-608.

Wooller, R., \& Brown, A. R. (2005). Investigating morphing algorithms for generative music. In Proceedings of the International Conference on Generative Systems in the Electronic Arts.

Worth, P., \& Stepney, S. (2005). Growing music: Musical interpretations of L-Systems. In Proceedings of the Conference on Applications of Evolutionary Computation, pp. $545-550$.

Yi, L., \& Goldsmith, J. (2007). Automatic generation of four-part harmony. In Proceedings of the Conference on Uncertainty in Artificial Intelligence.

Yilmaz, A. E., \& Telatar, Z. (2010). Note-against-note two-voice counterpoint by means of fuzzy logic. Knowledge-Based Systems, 23(3), 256-266.

Zicarelli, D. (1987). M and Jam factory. Computer Music Journal, 11(4), 13-29.

Zimmermann, D. (2001). Modelling musical structures. Constraints, 6(1), 53-83. 